\title{
Atmospheric Predictability of the Tropics, Middle Latitudes, and Polar Regions Explored through Global Storm-Resolving Simulations
}

\author{
FALKO JUDT \\ National Center for Atmospheric Research, Boulder, Colorado
}

(Manuscript received 2 May 2019, in final form 16 October 2019)

\begin{abstract}
The predictability of the atmosphere has important implications for weather prediction, because it determines what forecast problems are potentially tractable. Even though our general understanding of error growth and predictability has been increasing, relatively little is known about the detailed structure of atmospheric predictability, such as how it varies between climate regions. The present study addresses this issue by exploring error growth and predictability in three latitude zones, using model output from a previous global storm-resolving predictability experiment by Judt published in 2018. It was determined that the tropics have longer predictability than the middle latitudes and polar regions (tropics, $>20$ days; middle latitudes and polar regions, a little over 2 weeks). Each latitude zone had distinct error growth characteristics, and error growth was broadly consistent with the underlying dynamics of each zone. Evidence suggests that equatorial waves play a role in the comparatively long predictability of the tropics; specifically, equatorial waves seem to be less prone to error growth than middle-latitude baroclinic disturbances. Even though the generality of the findings needs to be assessed in future studies, the overall conclusions agree with previous work in that current numerical weather prediction procedures have not reached the limits of atmospheric predictability, especially in the tropics. One way to exploit tropical predictability is to reduce model error, for example, by using global storm-resolving models instead of conventional models that parameterize convection.
\end{abstract}

\section{Introduction}

Recently, Judt (2018) employed a global stormresolving model ${ }^{1}$ to revisit the issue of atmospheric predictability. The present study expands on the globalmean approach of Judt (2018) by exploring the predictability of three climate zones, namely, the tropics, middle latitudes, and polar regions. Following the theme of Judt (2018), this study addresses fundamental aspects of predictability rather than the more practical issue of forecast skill. Nevertheless, this study may still provide some value for the broader weather prediction community, because the predictability limits documented herein establish upper bounds for numerical weather prediction.

Earth's atmosphere is broadly organized into latitude zones, and the flow in each zone is governed by distinct

\footnotetext{
${ }^{1}$ Also referred to as global convection-permitting model or global cloud-resolving model.
}

Corresponding author: Falko Judt, fjudt@ucar.edu dynamics. For example, the flow in the middle latitudes is predominantly associated with baroclinic instability, whereas the flow in the tropics is primarily associated with moist convection. Scientists generally accept that error growth is a function of the underlying dynamics; therefore, one may expect that predictability is latitude dependent. Yet despite this argument, researchers often investigate error growth and predictability solely in the context of global means (e.g., Lorenz 1982; Boer 1994; Tribbia and Baumhefner 2004; Judt 2018)—a shortcoming that motivates the present study.

For practical purposes, operational centers have been routinely monitoring the skill of numerical models for the middle latitudes (e.g., Simmons and Hollingsworth 2002; Bengtsson and Hodges 2006; Buizza and Leutbecher 2015). Those reports, in conjunction with theoretical studies such as Lorenz (1969), led to the conclusion that middle-latitude flow is predictable for about 2 weeks or a little longer-a time frame that seems to hold even for the latest generation of global models (Zhang et al. 2019; Selz 2019). The consistency across model generations provides confidence in the 2-week limit, and it indicates that the limit of middle-latitude predictability is 
determined by errors growing in well-resolved baroclinic disturbances.

Estimating the predictability of the tropics turned out to be more challenging, in part because current global models cannot properly resolve moist convection. Traditionally, global models parameterize convection, and consequently, it is difficult to separate errors that grow due to model inadequacies from errors that grow due to internal instabilities (Reynolds et al. 1994). An additional issue is that error growth is unrealistically slow in models with parameterized convection (Selz and Craig 2015).

The present study bypasses these problems by explicitly modeling convection. It furthermore complements the work of Mapes et al. (2008), who investigated tropical error growth through storm-resolving aquaplanet simulations. Mapes et al. (2008) found that largescale errors had not saturated by day 16 , implying that tropical predictability reaches beyond the 2 -week limit of the middle latitudes. Straus and Paolino (2008) used a much lower resolution general circulation model, but arrived at similar conclusions; in fact, their results suggest that the tropical atmosphere is predictable for at least 1 month.

The reason behind the longer predictability of the tropics remains unclear. One hypothesis is that convectively coupled equatorial waves are more resistant to error growth than baroclinic systems. However, except for a recent study by Ying and Zhang (2017), the predictability of equatorial waves has neither received much attention nor has it been weaved into a general theory of tropical predictability.

Our understanding of the predictability of the polar atmosphere remains incomplete as well. One may argue that polar predictability on weather time scales is similar to that of the middle latitudes because the flow in both zones is dominated by baroclinic instability. At a closer look, however, the polar atmosphere is distinct. For example, the polar atmosphere is practically devoid of moist convection - the initial driver of error growth (e.g., Zhang et al. 2003, 2007; Judt 2018). Furthermore, the polar atmosphere gives rise to unique features such as tropopause polar vortices (TPVs), which have much longer lifetimes than baroclinic lows and may therefore have longer predictability (Cavallo and Hakim 2009, 2010). Last, events such as sudden stratospheric warmings may temporarily extend the predictability of the polar atmosphere to subseasonal and seasonal time scales (e.g., Baldwin and Dunkerton 2001; Thompson et al. 2002; Tripathi et al. 2016). Needless to say, the predictability of the polar atmosphere deserves to be explored in greater depth.

In short, the main goal of the present study is to further our understanding of atmospheric predictability.
The novelties lie in 1) the use of global model that explicitly simulates convection and 2) the contrasting juxtaposition of tropical, middle-latitude, and polar predictability. Naturally, this study has several limitations. First, it is essentially a case study, for multiple experiments of this sort would require immense computational resources. Second, the experiment was conducted with an atmosphere-only model, disregarding feedbacks between the atmosphere and other Earth system components. Third, error growth and predictability were measured with classic grid point metricsuseful for quantifying the predictability of turbulent flows but not ideal for assessing the predictability of individual weather events and other coherent phenomena.

The paper is organized as follows: section 2 briefly describes the data and methods. Sections 3 and 4 present the error growth and predictability analysis in physical and spectral space, respectively. Section 4 also offers a closer look at atmospheric kinetic energy (KE) spectra, scale-dependent predictability limits, and reveals possible reasons for some unexpected predictability behavior documented in Judt (2018). The predictability of TPV-like vortices is discussed in section 5, while 6 focuses on the predictability equatorial waves. A summary and conclusions follows in section 7 .

\section{Data and methods}

The present study is based on the identical-twin predictability experiment of Judt (2018) and uses the same model output. Therefore, only a brief experiment overview will be provided here. See section 2 of Judt (2018) for more details.

The experiment comprises three sets of identical twins with lengths of 20,15 , and 10 days, respectively, realized by integrating a 20-day-long control simulation (0000 UTC 20 October-9 November 2012) and three perturbed simulations that were started at 0000 UTC 20, 25, and 30 October 2012, respectively (Judt 2018, his Fig. 1). As usual, the "error" was defined as the difference between control and any of the perturbed runs. The simulations were produced with the Model for Prediction Across Scales (MPAS; Skamarock et al. 2012) on a globally uniform mesh with 4-km cell spacing. This grid spacing is "convection permitting," and a classic convection parameterization was not used ${ }^{2}$.

Atmospheric predictability was explored in three regions: tropics $\left(10^{\circ} \mathrm{S}-10^{\circ} \mathrm{N}\right)$, middle latitudes $\left(35^{\circ}-50^{\circ} \mathrm{N} / \mathrm{S}\right)$,

\footnotetext{
${ }^{2}$ The model was set up with the "scale-aware" convection scheme of Grell and Freitas (2014), which generated 15\% of the overall precipitation.
} 


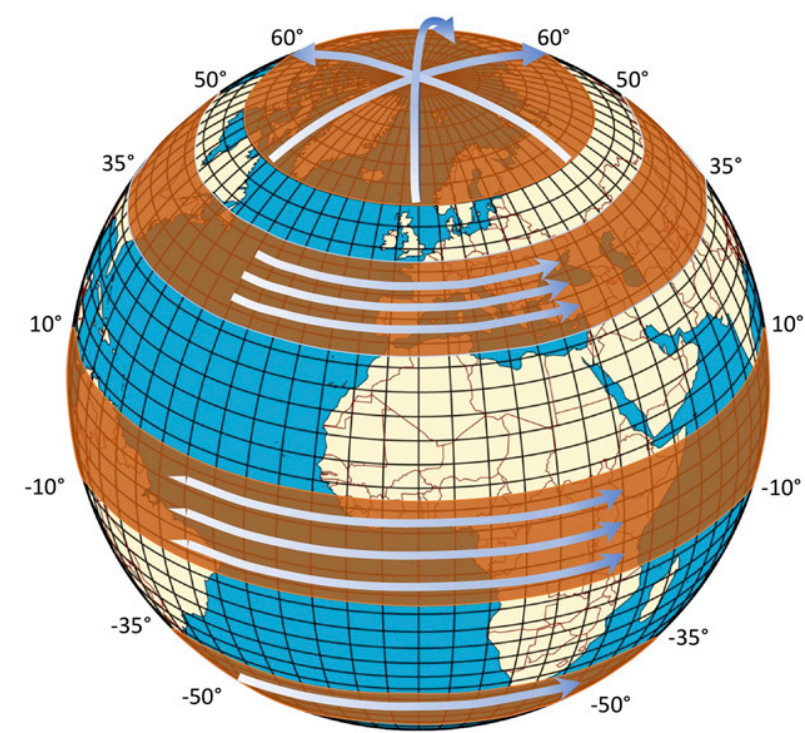

FIG. 1. Latitude zones in which error growth and predictability are analyzed (brown shading; tropics: $10^{\circ} \mathrm{S}-10^{\circ} \mathrm{N}$; middle latitudes: $35^{\circ}-50^{\circ} \mathrm{N} / \mathrm{S}$; polar regions: poleward of $60^{\circ} \mathrm{N} / \mathrm{S}$ ). The arrows indicate the direction in which fields are Fourier-decomposed to obtain KE and Z500 spectra, i.e., in the tropics and middle latitudes, fields are decomposed in longitude, but in the polar regions, fields are decomposed in latitude.

and polar regions (poleward of $60^{\circ} \mathrm{N} / \mathrm{S}$; Fig. 1). To increase the number of data samples and reduce noise, the middlelatitude bands and polar caps of each hemisphere were combined into a single domain (interhemispheric differences can therefore not be discussed).

The error was first quantified with two standard metrics, difference kinetic energy (DKE) and root-mean-square error of 500-hPa geopotential height (Z500 RMSE $)$. DKE and $\mathrm{Z} 500_{\mathrm{RMSE}}$ were computed for each latitude zone according to

$$
\operatorname{DKE}(p, t)=\frac{1}{2} \overline{\left(\Delta u^{2}+\Delta v^{2}\right)}
$$

and

$$
\mathrm{Z} 000_{\mathrm{RMSE}}(t)=\sqrt{\overline{\left(\Delta Z_{500}\right)^{2}}} .
$$

In the above equations, $u$ is zonal wind, $v$ is meridional wind, Z500 is 500-hPa geopotential height, $\Delta$ indicates the difference between control and perturbed run (i.e., the error), and the overbar denotes an average of all grid points in a latitude zone. The independent variables $p$ and $t$ are pressure and time, respectively. DKE was computed on pressure levels of 925, 850, 700, 500, 250, and $200 \mathrm{hPa}$.

Since the error saturates at twice the climatological variance, the saturation limits were determined from reanalysis fields as follows:

$$
\begin{aligned}
& \operatorname{Limit}_{\mathrm{DKE}}(p)=\frac{1}{61} \sum_{d=1}^{61} 2[\overline{\operatorname{var}(u)+\operatorname{var}(v)]}, \\
& \text { Limit }_{\mathrm{Z500} \text { RMSE }}=\frac{1}{61} \sum_{d=1}^{61} \sqrt{\frac{2 \operatorname{var}(\mathrm{Z} 500)}{}} .
\end{aligned}
$$

Here $\operatorname{var}(u), \operatorname{var}(v)$, and $\operatorname{var}(\mathrm{Z} 500)$ are the gridpoint variances computed over the 30-yr period 1987-2016. The overbar again denotes an average of all grid points in a latitude zone. Sixty-one variance averages were computed, one for each day in October and November. These 61 values were then averaged to obtain a single value representative of the experiment period. Saturation limits were computed from both ERA-Interim (ERA-I) and the newer ERA5 dataset. We will see in section 3 that the magnitude of the saturation limit depends on whether one uses ERA-I or ERA5 data.

As usually done in predictability studies, the scale dependency of predictability was explored via spectral decomposition. Specifically, the $u, v$, and Z500 fields were first interpolated to a regular latitude-longitude mesh with $0.01818^{\circ}(\sim 2 \mathrm{~km})$ resolution. Then, the wind and height fields as well as their respective difference fields were Fourier decomposed. In the tropics and middle latitudes, fields were Fourier decomposed in longitude, but in the polar regions, fields were decomposed in latitude to circumvent the problem of converging meridians at the poles (Fig. 1). Following Errico (1985), the linear trend of $u, v$, and Z500 on meridians across the poles was removed to enforce periodicity. After the Fourier decomposition, a coordinate transform was applied to map each 1D power spectrum from wavenumber into wavelength space. This step is convenient for two reasons: first, wavelength better conforms to the normal meaning of scale, and second, spectra can be averaged in latitude without losing the ability to attribute a physical wavelength to a given wavenumber. (The polar spectra obtained with the above-described method are equivalent to spectra obtained by rotating the globe and placing the polar caps on the equator and applying Fourier analysis in longitude on square meshes with similar size to the polar caps.)

For the equatorial wave analysis, model fields were filtered in the Wheeler-Kiladis wavenumber-frequency domain (Wheeler and Kiladis 1999). Specifically, precipitation, 850-hPa zonal wind, and 850-hPa meridional wind were first interpolated to a regular latitude-longitude grid with $0.25^{\circ}$ resolution at 6-hourly intervals. To focus on the main region of wave activity, only data between $10^{\circ} \mathrm{N}$ and $10^{\circ} \mathrm{S}$ were retained. The clipped fields were averaged in the meridional direction to yield time-longitude arrays, and subsequently filtered with the NCAR Command Language "kf-filter" function to extract the waves. 

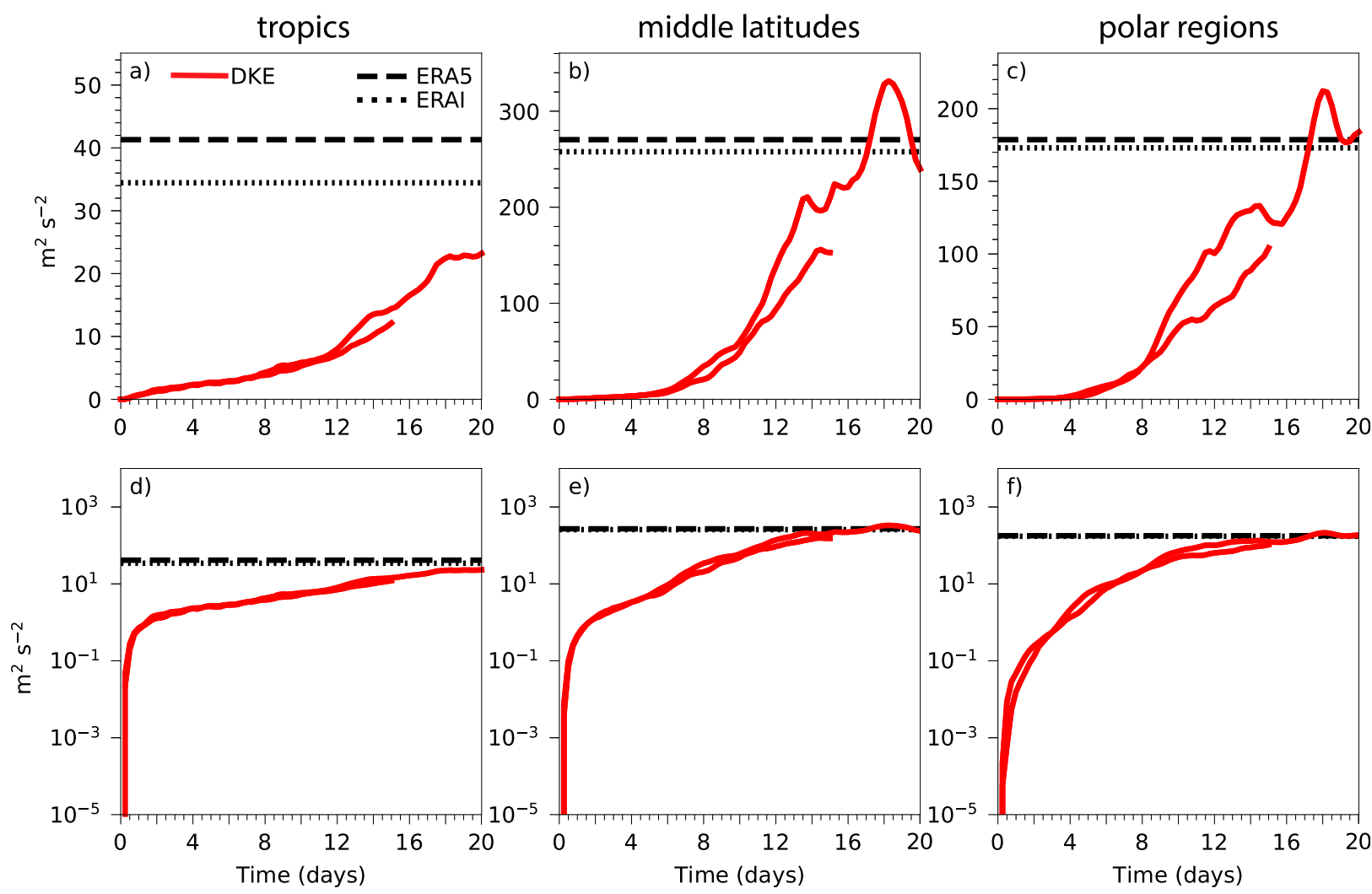

FIG. 2. (top) Time series of volume-averaged DKE for the (a) tropics, (b) middle latitudes, and (c) polar regions. Black lines denote the saturation limits computed from ERA5 (dashed) and ERA-I (dotted). (bottom) As in the top row, but with a logarithmic $y$ axis. Each panel has two DKE graphs with lengths of 20 and 15 days (the 10-day experiment has been omitted for clarity).

The kf-filter algorithm assumes that the underlying data are periodic in time, and to alleviate artifacts from this assumption being violated, the time-longitude arrays were padded with missing values prior to filtering. Specifically, the 20-day runs were padded with 10 days of missing values, whereas the 15- and 10-day runs were padded with 15 and 20 days of missing values, respectively.

\section{Error growth and predictability in physical space}

Let us begin the predictability analysis by considering the evolution of volume-averaged DKE, a convenient metric to quantify the bulk error in atmospheric flow (Fig. 2). Following Judt (2018), time series of DKE are presented in plots with linear (Fig. 2, top) and logarithmic $y$ axis (Fig. 2, bottom). Figure 2 conspicuously tells that the predictability of the tropics exceeds that of the extratropics. Specifically, the tropical atmosphere seems to be predictable beyond 20 days, for the error does not even come close to saturation during the 20-day period (Fig. 2a). In contrast, error saturation occurs on approximately day 17 in the middle latitudes and polar regions (Figs. 2b,c).
These results are robust even when recognizing that the saturation limit depends on the choice of reanalysis (horizontal dashed lines in Fig. 2). The discrepancy between ERA-I and ERA5 is particularly prominent in the tropics, where the ERA5 saturation limit is almost $20 \%$ higher than its ERA-I counterpart (Fig. 2a). Evidently, the wind variance is higher in ERA5, possibly because the higher-resolution ERA5 includes the variance of motions that ERA-I cannot resolve. Given that ERA5 supersedes ERA-I (Hersbach and Dee 2016), ERA5 data are deemed more accurate here, and only the ERA5 saturation limit will be considered from here on.

Error growth seems to be broadly consistent with the underlying dynamics of each zone. Consider the time between 4 and 20 days, a period during which error growth is not only qualitatively similar in the middle latitudes and polar regions (Figs. 2b,c), but also quantitatively, as revealed by comparable error doubling times (Table 1). This similarity is consistent with errors growing through similar processes in those regions, probably baroclinic instability. A different process must be at work in the equatorial regions, where the error 
TABLE 1. Error (DKE) doubling times, computed according to Eq. (4) from Judt (2018) with 6-hourly data. The values in the second column are an average of the 28 error doubling time values in the 7-14-day period. Negative doubling times mean that the error magnitude decreases with time.

\begin{tabular}{lrr}
\hline \hline & $0-6 \mathrm{~h}$ & $7-14$ days \\
\hline Tropics & $<1 \mathrm{~h}$ & $131 \mathrm{~h}$ \\
Middle latitudes & $1 \mathrm{~h}$ & $56 \mathrm{~h}$ \\
Polar regions & $-3 \mathrm{~h}$ & $53 \mathrm{~h}$ \\
\hline
\end{tabular}

growth rate is smaller. In fact, the error doubling time in the tropics is more than twice as large as in the other two zones (Table 1).

When focusing on the first 2 days instead of days 4-20, the relationship reverses in that middle-latitude error growth resembles that in the tropics rather than that in the polar regions. Specifically, tropics and middle latitudes feature the telltale sign of error growth through moist convection, that is, an initial burst of explosive growth (error doubling times of $\leq 1 \mathrm{~h}$; Table 1 ), followed by a distinct transition to slower growth (Figs. 2d,e). The polar regions, which lack convection, show much less of a distinct transition (Fig. 2f). During the first hour, the error even reduces in the polar region, followed by slow growth. As a result, the error doubling time for the 0-6-h period is negative (Table 1 ).

One question that Fig. 2 cannot answer is whether predictability is height dependent. It turns out that, broadly speaking, predictability has little systematic height dependence, except that the normalized error tends to decrease with height (Fig. 3). In the polar regions, the DKE time series are essentially congruent (Fig. 3, right column). In the tropics (Fig. 3, left column), the graphs look least congruent, and at day 20 , the error fraction decreases from near $70 \%$ at $850 \mathrm{hPa}$ to about $50 \%$ at $200 \mathrm{hPa}$ (Fig. 3a). The middle latitudes show a behavior somewhere in between the tropics and polar regions.

Besides the comparatively more pronounced height dependence, tropical error growth seems to be distinct in another way. Around 10-12 days, the tropical error growth rate increases, especially in comparison with the more gradual increase of the growth rate in the middle latitudes and polar regions. This point is hardly noticeable in Figs. 2 and 3, but it stands out in the Z500 ${ }_{\text {RMSE }}$ time series where there is a clear "kink"
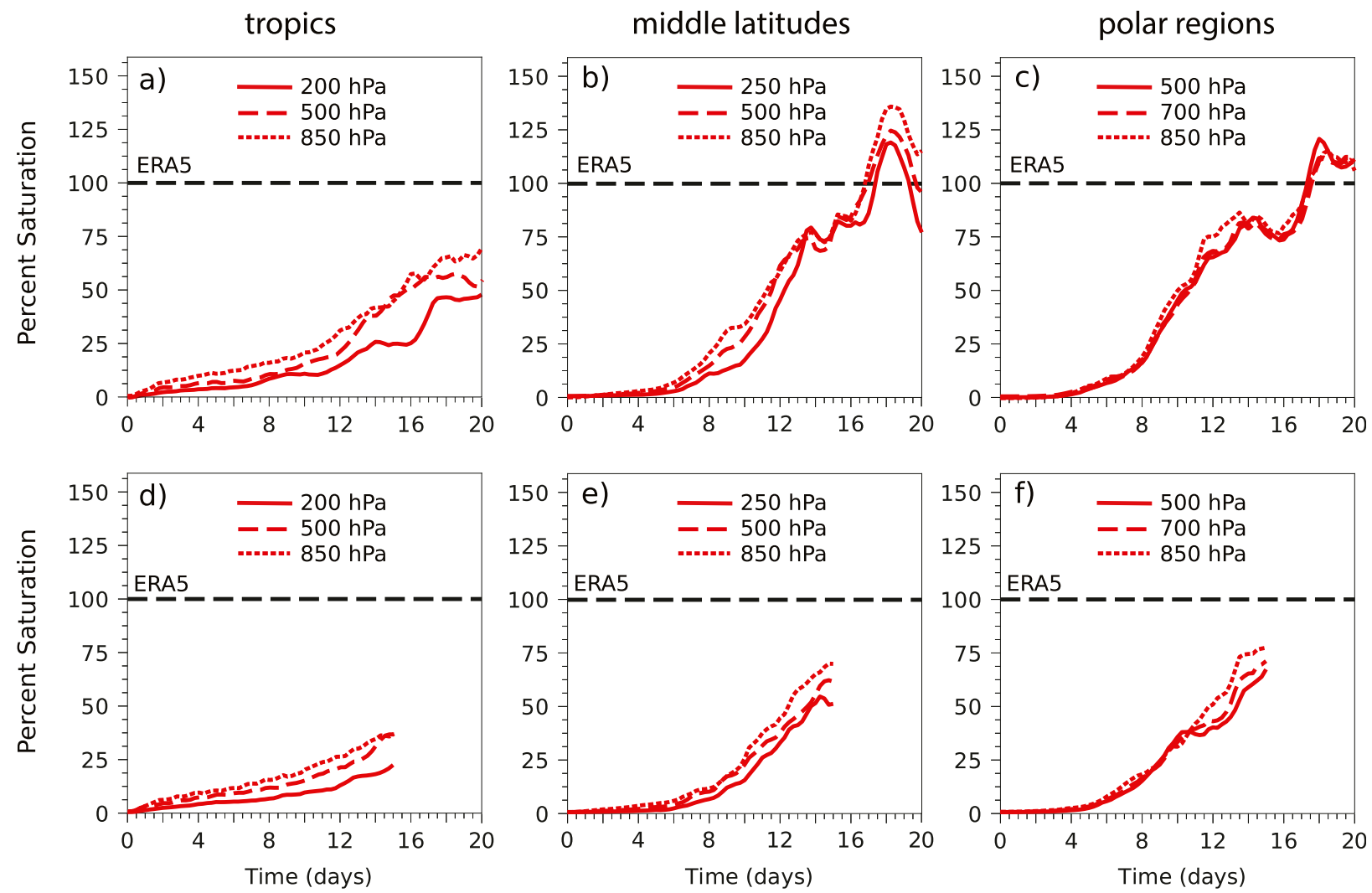

FIG. 3. As in Fig. 2, but instead of volume averages, the panels show horizontal averages at three levels: upper troposphere (solid lines), middle troposphere (dashed lines), and lower troposphere (dotted lines). The DKE time series are normalized by the respective saturation limits. (top) The 20-day runs and (bottom) the 15-day runs. 


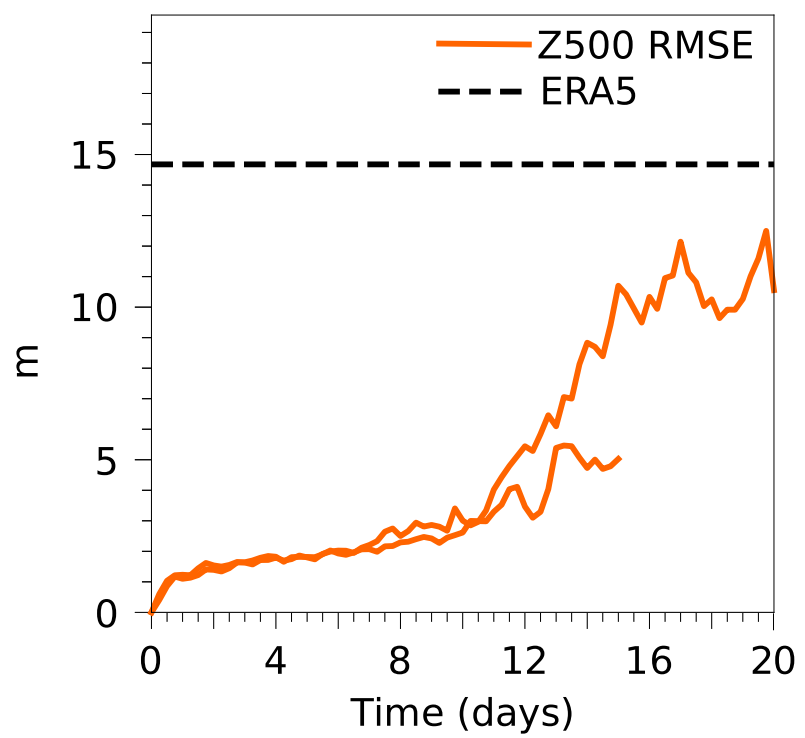

FIG. 4. Time series of Z500 ${ }_{\text {RMSE }}$ averaged over the tropics. The dashed line denotes the saturation limit computed from ERA5. There are three Z500 RMSE graphs with lengths of 20 and 15 days (the 10-day experiment has been omitted for clarity).

between two phases of quasi-linear growth (Fig. 4). One may certainly question the importance and robustness of this finding given the limited sample size, but section 4 will show that an increase in growth rate around day 10 is consistent with error growth through equatorial waves.

In summary, this section revealed that error growth and atmospheric predictability are functions of latitude zone, and that error growth is generally consistent with the underlying dynamics of each zone. The predictability limit of the middle latitudes was determined to be a little over 2 weeks, a time span that agrees with other studies to within a day or two (e.g., Simmons and Hollingsworth 2002; Zhang et al. 2019; Selz 2019). The polar regions seem to have a similar predictability horizon, although errors initially grow much more slowly. The predictability limit of the tropics could not be specified; however, it seems to be substantially longer than that of the extratropics ( $>20$ days). This finding is in accord with Straus and Paolino (2008).

\section{Error growth and predictability in spectral space}

This section has three main objectives, the first of which is to investigate the latitude dependence of the atmospheric KE spectrum (also referred to as the background spectrum). The second objective is to quantify the scale-dependent predictability limits in each latitude zone, and the third is to reveal possible reasons for some unexpected predictability behavior documented in Judt (2018).

As in the previous section, we will first discuss vertically averaged quantities, beginning with the background spectrum (Fig. 5). In general, the slope of the background spectrum steepens as one moves from the equator toward the poles. Specifically, the tropics have a relatively shallow spectrum with a quasi-uniform slope close to $-5 / 3$ (Fig. 5a). The middle latitudes, shown in Fig. 5b, feature a canonical two-segment spectrum with a slope near -3 at the synoptic scales (wavelengths around $1000 \mathrm{~km})$ and a slope near $-5 / 3$ at the mesoscales (wavelengths $<300 \mathrm{~km}$ ). Finally, the polar regions have a steeper spectrum with a slope is closer to
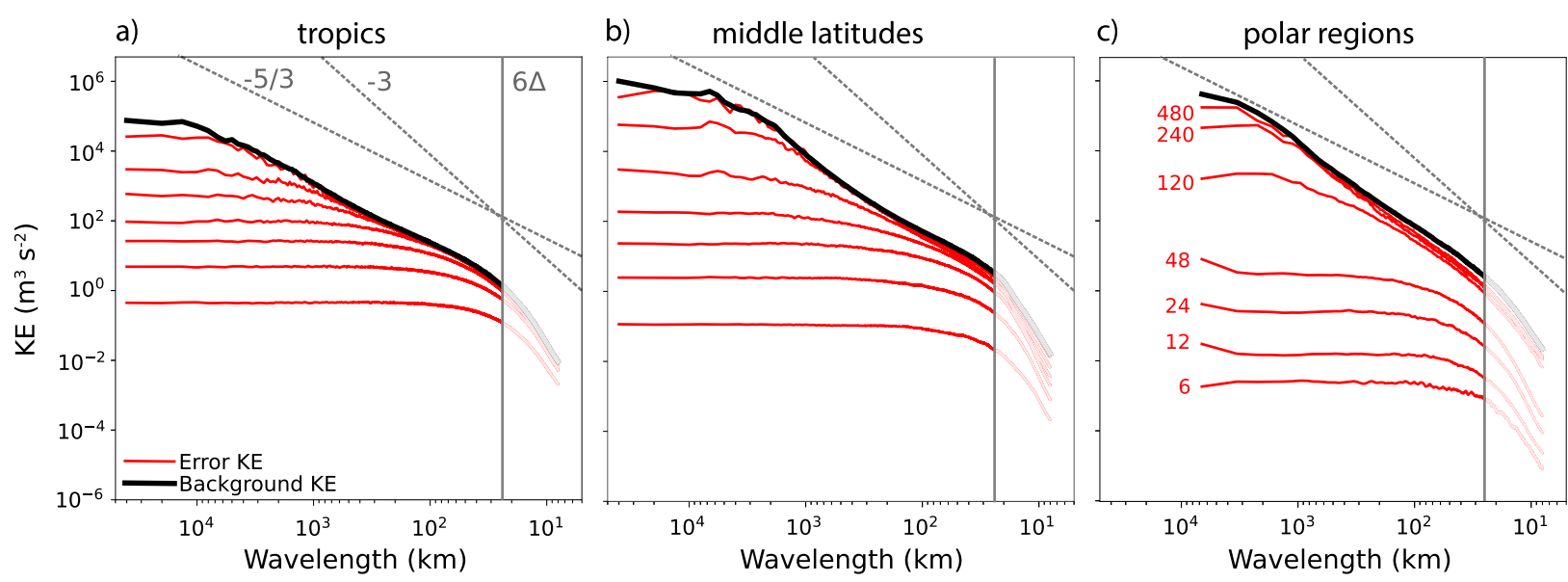

FIG. 5. Background KE spectra (black) and error KE spectra (red), vertically averaged over the (a) tropics, (b) middle latitudes, and (c) polar regions. Error spectra are valid at 6,12, 24, 48, 120,240, and $480 \mathrm{~h}$, as indicated by the red numbers in (c). Background spectra are time-mean ensemble means multiplied by 2 to highlight the saturation limit. Error spectra are averaged over the number of experiments available at a given time. For reference, each panel includes graphs with logarithmic slopes of -3 and $-5 / 3$ (dotted lines). Data beyond the effective resolution limit of $24 \mathrm{~km}$, which is indicated by the vertical gray lines, are not considered. 
-3 and only a little bit of shallowing at the mesoscales (Fig. 5c).

The fact that the spectral slope differs between the three zones is almost certainly a consequence of the underlying dynamics. For example, the $k^{-5 / 3}$ spectrum in the tropics is consistent with a convecting flow that lacks broad rotational motions [according to Sun et al. (2017) and Weyn and Durran (2017) moist convection is sufficient to produce a $k^{-5 / 3}$ spectrum]. The flow in the middle latitudes contains broad rotational motions such as planetary Rossby waves as well as intermittent and localized convection, a combination that manifests in the canonical spectrum with slopes of -3 and $-5 / 3$. The large-scale flow in the polar regions is also dominated by broad rotational motions, but in contrast to the middle latitudes, the lack of convection precludes the formation of a mesoscale $k^{-5 / 3}$ spectrum. The weak shallowing in the polar regions may be associated with topographically induced gravity waves (Menchaca and Durran 2019).

Next, we will examine the error spectra in Fig. 5. In contrast to the sloped error spectra of Judt (2018), which were obtained via spherical harmonics transform, the Fourier-derived error spectra in Fig. 5 have the form of horizontal lines akin to the similarly derived ones in Durran and Gingrich (2014) and Zhang et al. (2019). Other than the horizontal nature of the error spectra, their evolution generally agrees with previous studies. In particular, the error grows "up-magnitude" rather than "up-scale" (e.g., Mapes et al. 2008; Durran et al. 2013; Durran and Gingrich 2014; Judt 2018).

In the tropics, the mesoscale error quickly approaches the background spectrum (which marks the saturation limit). For example, at wavelengths $<100 \mathrm{~km}$, the error approaches the saturation limit within $24 \mathrm{~h}$ (Fig. 5a). In the middle latitudes, this process takes substantially longer, and for the same scales, the error does not approach the saturation limit before $48 \mathrm{~h}$ (Fig. $5 \mathrm{~b}$ ). The error amplitude in the polar regions is initially much smaller than in either other zone and reaches only about $10 \%$ saturation at the smallest resolved scales by $48 \mathrm{~h}$.

As the error grows, the error spectra close in on the saturation limit at successively larger scales. By $480 \mathrm{~h}$ (20 days), the error in the tropics has saturated at scales up to about $5000 \mathrm{~km}$ (Fig. 5a). The middle-latitude error has even saturated at all scales by that time (Fig. 5b; the lack of saturation at certain wavelengths is interpreted here as an artifact of noise and limited sample size). This behavior signifies a total loss of middle-latitude predictability by day 20; an identical result was obtained by the ECMWF-model-based predictability study of Zhang et al. (2019). Even though the polar error is initially much smaller, its amplitude and saturation behavior become comparable with that of the middle latitudes at later times (Fig. 5c).

The differences and similarities in spectral error between the three zones reflect once more that error growth depends on the underlying dynamics. In particular, convection is key to initial error growth (tropics, middle latitudes), whereas baroclinic processes lead to higher growth rates later on (middle latitudes, polar regions).

The error spectra in Fig. 5 are valuable for revealing general properties of error growth, however, they are not ideal for quantitative analyses. Notably, the logarithmic nature of plots makes it is difficult to assess the precise time at which the error saturates because a seemingly negligible separation between error and background may actually be a substantial amount of "predictable energy." To assess the actual limits of predictability more precisely, the method of Judt (2018) was applied. Specifically, the predictability limit of a given wavelength was calculated by determining the forecast time at which the error reaches $90 \%$ saturation (Fig. 6; also shown is the time at which the error reaches $60 \%$ saturation, which is often considered the limit of useful prediction skill).

The data points in Fig. 6 trace a distinct pattern for each climate zone, meaning that each zone has particular predictability characteristics. For example, the predictability of mesoscale motions with scales $<100 \mathrm{~km}$ is shorter in the tropics than in the middle latitudes (5-7 vs 10-12 days), in agreement with Fig. 5. For synoptic-scale motions (wavelengths $>600 \mathrm{~km}$ ), this relationship reverses; in fact, while scales $>5000 \mathrm{~km}$ in the middle latitudes have predictability limits of 15-17 days, the error in the tropics never even reaches $60 \%$ saturation at those scales. The predictability of the polar regions is peculiar in that mesoscale errors do not saturate, although they reach $60 \%$ saturation well before day 10 .

The pattern traced by the orange data points is less noisy than that of their red counterparts, and since a threshold of $60 \%$ is often used to define useful prediction skill, the orange points may be the more useful ones for the weather prediction community. In this sense, skillful 5-day forecasts of mesoscale weather systems seem to be futile in the tropics, but certainly possible in the polar regions. On the other hand, weather systems with scales of $1000 \mathrm{~km}$ seem to have similar predictability horizons in all zones, roughly $10 \pm 2$ days.

Figure 6 raises an interesting question: Why does mesoscale flow have a shorter predictability time limit in the tropics than elsewhere? One possible reason is the lack of synoptic-scale forcing in the tropics, or in other words, convection and associated mesoscale motions are unconstrained (i.e., random and less predictable) in the tropics, but forced and constrained by the more 

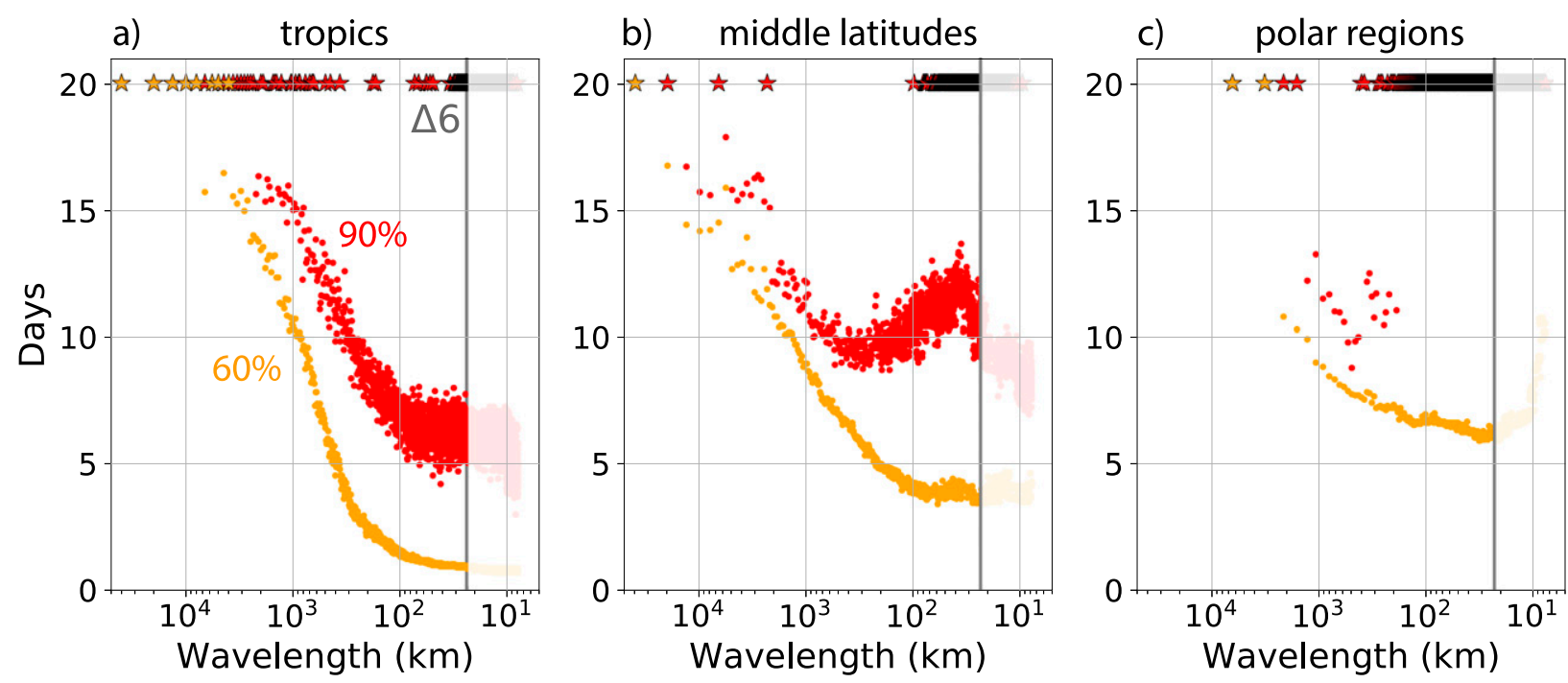

FIG. 6. Predictability limits ( $90 \%$ saturation; red dots) and limits of useful prediction skill ( $60 \%$ saturation; orange dots) as a function of scale. Red star symbols at the 20-day mark indicate that the $90 \%$ threshold has not been reached within the 20-day period (orange symbols mean the same, but for $60 \%$ saturation). Data beyond the effective resolution limit of $24 \mathrm{~km}$, which is indicated by the vertical gray lines, are not considered.

predictable synoptic-scale flow in the extratropics. More research is needed to test this hypothesis.

The next step in our analysis is to examine the height dependence of spectra and predictability (Figs. 7 and 8). Generally, the slopes of the background spectra steepen with height, a behavior that is especially apparent in the polar regions and the middle latitudes (Fig. 7, right two columns). Furthermore, the kink that links the $k^{-3}$ and $k^{-5 / 3}$ parts of the middle-latitude spectrum becomes less pronounced toward the surface, in agreement with the globally averaged spectra of Judt (2018).

Even though spectral error growth does not show any obvious dependence on height (Fig. 7), the scaledependent predictability limits in Fig. 8 show substantial variability with height, in addition to their variability across latitude zones. Evidently, atmospheric predictability is a complex issue, and the predictability limit of atmospheric motions is not only a function of scale, but also of height and latitude.

To highlight a few differences between the zones, consider the upper troposphere (Fig. 8, top). In the tropics, predictability limits increase monotonically from smaller to larger scales, meaning that the smallest resolved scales have the shortest predictability. In contrast, the middle latitudes and polar regions feature a predictability minimum at intermediate scales of $200-400 \mathrm{~km}$. When descending to the middle troposphere (Fig. 8, middle), the pattern in the middle latitudes aligns with that in the tropics; in particular, the relative maximum at the smallest resolved scales disappears (Fig. 8e). In the polar regions, predictability limits are generally longer, but there is no clear dependence on scale, except that many smaller scales do not saturate (Fig. 8f). Descending farther to the lower troposphere, the patterns become increasingly muddled by noise, especially in the tropics (Fig. 8, bottom).

Despite the complex and disorderly look of Fig. 8, there are a few commonalities between the three latitude zones. One commonality is that predictability limits generally increase as one descends toward the surface, especially at the mesoscales. For example, in the middle latitudes, the predictability limit of $100-\mathrm{km}$-scale motions is $<5$ days in the middle troposphere, but increases to $>10$ days in the lower troposphere (the middle latitudes are unique in that mesoscale predictability limits are longer at $250 \mathrm{hPa}$ than at $500 \mathrm{hPa}$, but it is unclear why this is the case).

Let us now turn our attention to the spectrum of Z500 (Fig. 9). In particular, we will compare the spectrum of Z500 power to that of 500-hPa KE. One similarity between the two spectra is the comparatively shallow slope in the tropics. Another similarity is that, in the middle latitudes, both spectra feature a pronounced kink between the mesoscale and synoptic-scale segments (Figs. 9a,b vs Figs. 7d,e). The two spectra differ in the polar regions; specifically, the kink is much more distinct in the Z500 spectrum compared to the 500-hPa KE spectrum (Fig. 9c vs Fig. 7c).

Menchaca and Durran (2019) showed that terraininduced gravity waves can generate a mesoscale $k^{-5 / 3}$ spectrum. We may therefore suspect that this mechanism plays a role in the pronounced shallowing of the mesoscale 

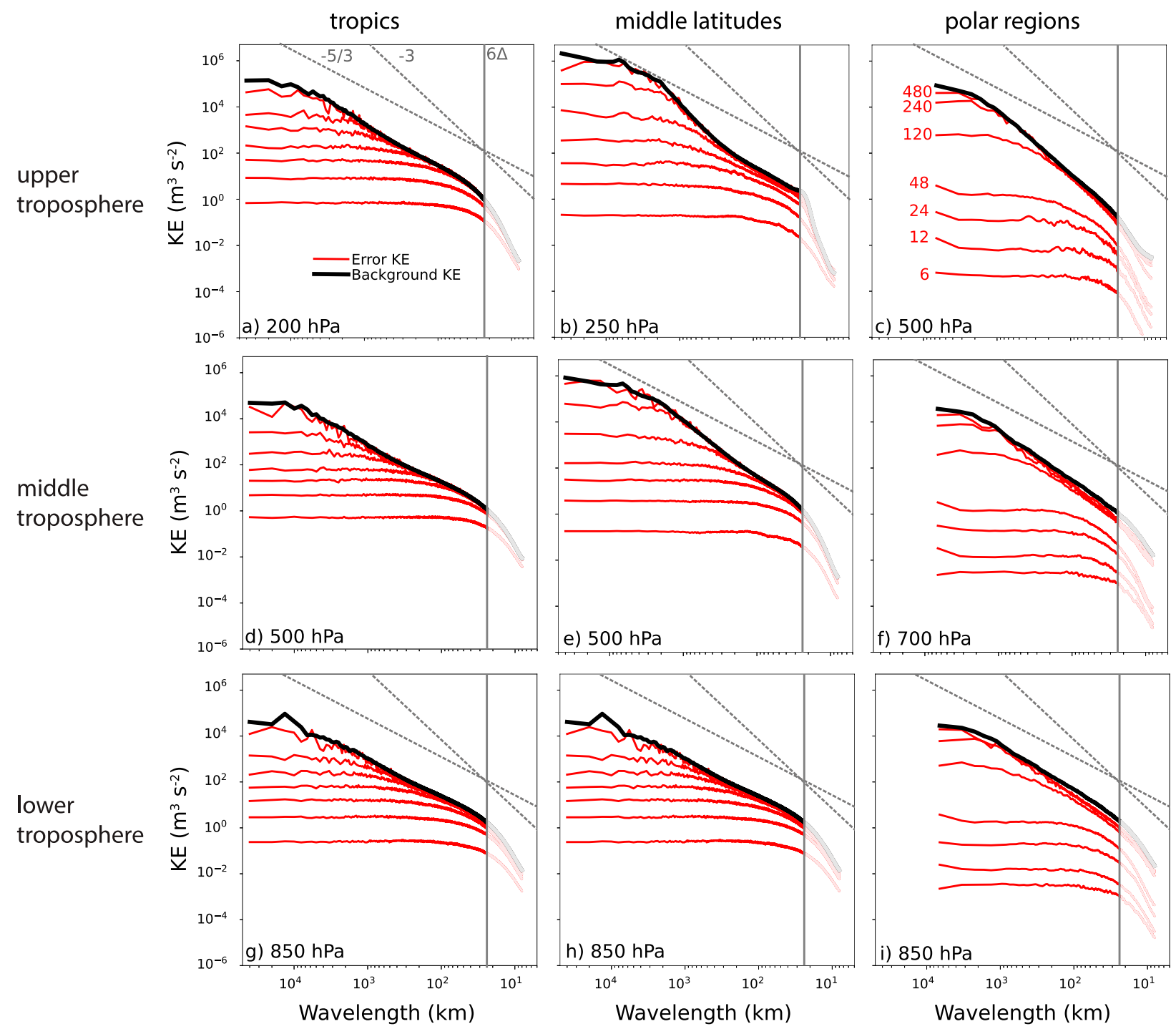

FIG. 7. As in Fig. 5, but instead of volume averages, spectra for various pressure levels are shown.

Z500 spectrum in the middle latitudes and the polar regions (Figs. 9b,c). What is not clear, however, is why this effect seems to have a lesser effect on the polar KE spectrum (Fig. 7, right column). Possibly, the terrain effect of Menchaca and Durran (2019) is not strong enough in these regions, as the mountain ranges in Earth's polar regions are lower and the mean westerly flow weaker than in the middle latitudes.

Judt (2018) noted a peculiarity in that Z500 error spectra did not saturate at wavelengths $<300 \mathrm{~km}$. This behavior is reproduced here, most notably so in the middle latitudes (Fig. 9b). Interestingly, the gap between background spectrum and 20-day error spectrum at the mesoscales disappears when we restrict the Z500 spectral analysis to a land-free region over the Pacific Ocean instead of full latitude circles (Fig. 10). Note that the mesoscale saturation over the South Pacific occurs because the background spectrum has less power at short wavelengths, not because the error spectrum has more power (one can compare the 20-day error spectra in Figs. 10 and $9 \mathrm{~b}$ and will notice the power is similar at the cutoff wavelength of $24 \mathrm{~km}$ ).

A question that naturally follows is, Why does the Z500 spectrum over the South Pacific have comparatively less power at the mesoscales? One hypothesis is that the extra power in the Z500 background spectrum in Fig. 9b is associated with shortwave components of standing disturbances over topography. The mesoscale errors are produced by vertically propagating terraininduced gravity waves whose signal shows up in velocity (leading to the saturation at short wavelengths in Fig. 7e), but not in Z500. These waves typically tilt 

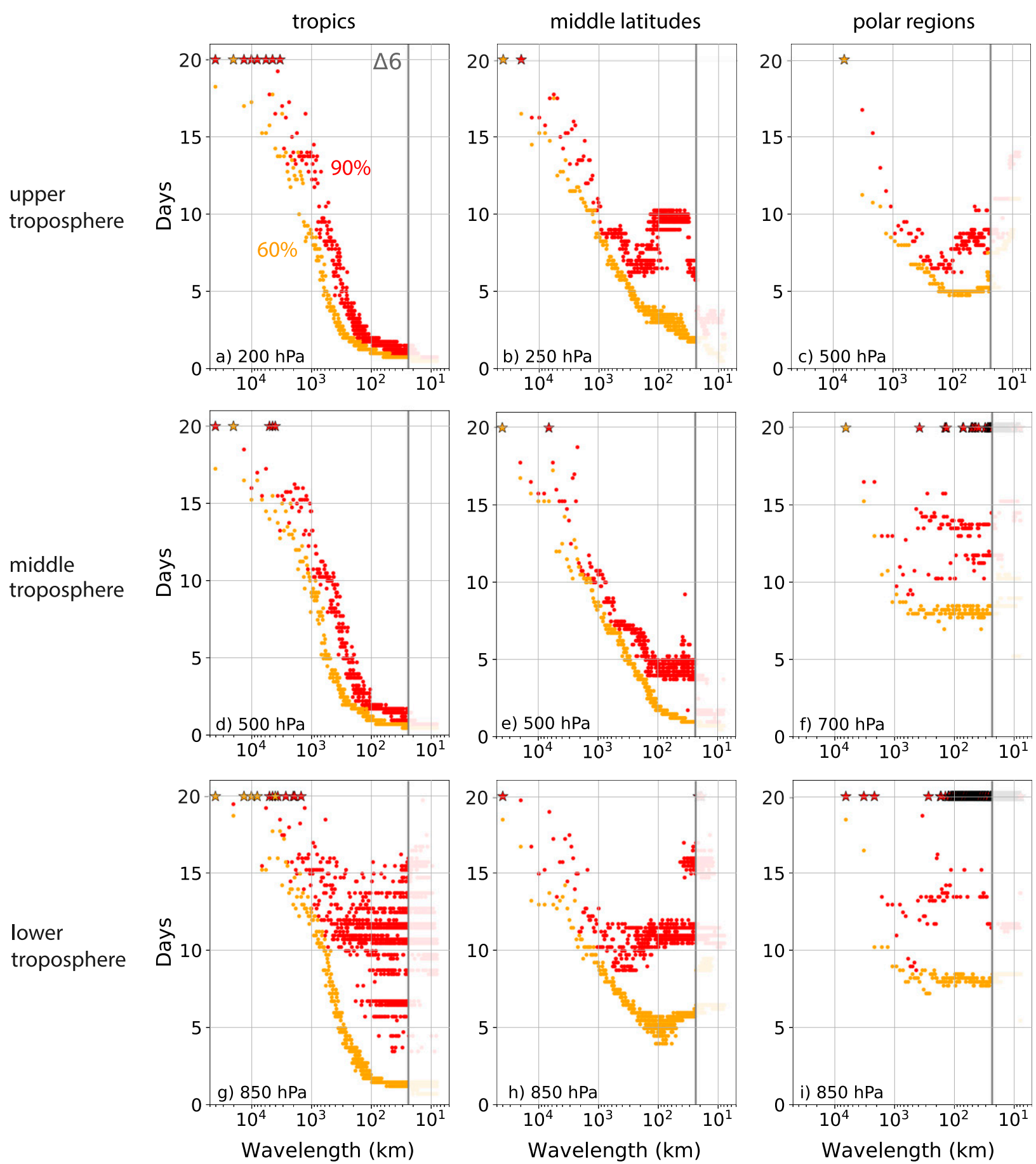

FIG. 8. As in Fig. 6, but instead of vertical averages, data for various pressure levels are shown.

strongly with height so that the vertical integral of their buoyancy perturbations will not have a large impact on the vertically integrated pressure perturbations and the height of the 500-hPa pressure surface.

The other unanticipated behavior noted by Judt (2018) was that predictability times increase toward the surface.
This behavior was also reproduced here (Fig. 8). One can speculate again that topography (or more generally, the land surface) causes this behavior, specifically, the land surface imposes structure on the flow that hinders decorrelation of the flow (a full decorrelation of eddies in the flow is equivalent to a complete loss of 

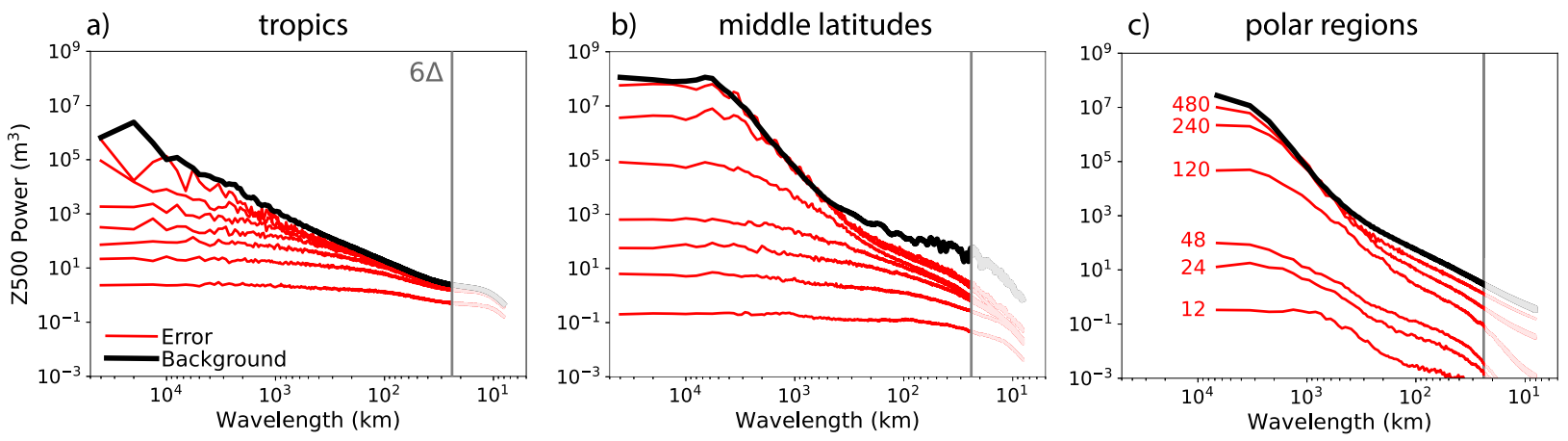

FIG. 9. Background spectrum (black) and error spectra (red) of Z500 power.

predictability). A comparison of Fig. 11 with the middle column of Fig. 8 supports this hypothesis: predictability limits seem to be less height dependent when computed for the land-free region (Fig. 11) than when computed for the entire middle-latitude belt (Fig. 8, middle column).

To summarize, this section revealed that the slope of the KE spectrum varies with latitude (and to a lesser degree with height). The predictability limit of atmospheric motions was determined to depend on scale, latitude, and height. Broadly speaking, mesoscale motions have shorter predictability in the tropics than in the extratropics, but the reverse is true for large-scale motions. Finally, effects of topography seem to be the reason for increase in predictability times toward the surface, especially for mesoscale motions.

\section{Predictability of TPV-like features in the polar atmosphere}

The previous sections showed that the extratropical flow loses predictability after a little over 2 weeks in this experiment. In the middle latitudes, the loss of predictability is a manifestation of decorrelating troughs and ridges in the Rossby wave pattern (Baumgart et al. 2019), a process that is illustrated in Fig. 12: at simulation day 15 , the control simulation features a wavy jet stream pattern with a ridge centered southwest of Ireland and a deep trough over Europe (Fig. 12a). On the other hand, the perturbed simulation features a zonal jet stream over northern Europe with two cutoff lows east and west of the Iberian Peninsula (Fig. 12b).

The snapshots of 500-hPa wind speed and vorticity in Fig. 12 also illustrate how the flow in the arctic differs from that in the middle latitudes. Specifically, the winds in the arctic are weaker, and the vorticity field is smoother and devoid of "noise" that appears in the middle latitudes. This noise, which is made up of smallscale granules with positive and negative vorticity, is mostly associated with frontal convection and flow over topography. One of the unique features of the polar atmosphere is the occurrence of tropopause polar vortices (TPVs), features that are frequently observed poleward of the middle-latitude jet streams (Cavallo and Hakim 2009, 2010). TPVs are smaller than typical baroclinic lows and have lifetimes that can exceed 1 month, and especially the latter fact suggests that TPVs may have long predictability.

Because of the predictability framework chosen for this study, which heavily relies on bulk metrics, it is difficult to assess the predictability of coherent entities such as TPVs. Nonetheless, an attempt is made here with the goal demonstrate that TPVs have relatively long predictability. First, we ensure that the simulations give rise to TPVs or TPV-like vortices. Some of the candidates are displayed in 13 ; they differ from vorticity features in the middle latitudes in that they are more compact and spatially coherent, they are relatively small scale (diameters of the vorticity "bull's-eyes"

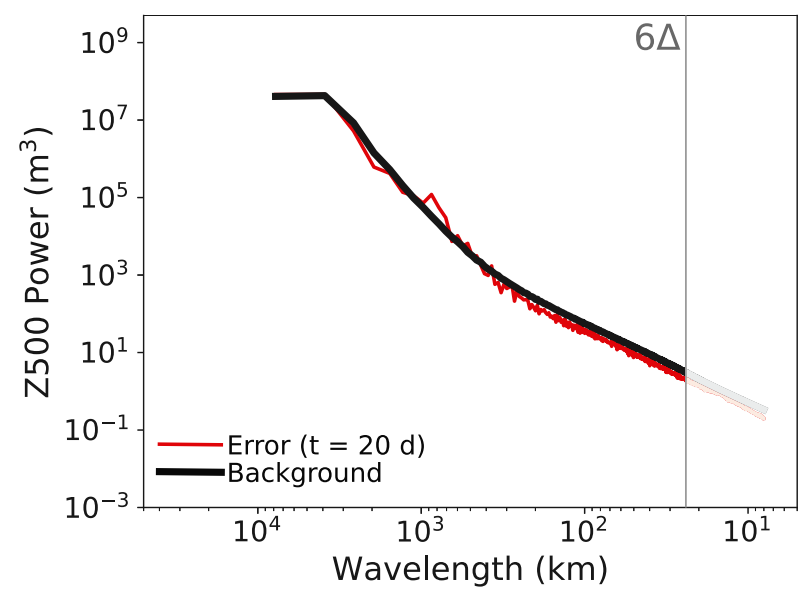

FIG. 10. Z500 power spectrum (black) and $t=20$-day Z500 error spectrum (red), computed over a land-free region over the South Pacific $\left(35^{\circ}-50^{\circ} \mathrm{S}, 85^{\circ} \mathrm{W}-180^{\circ}\right)$. 

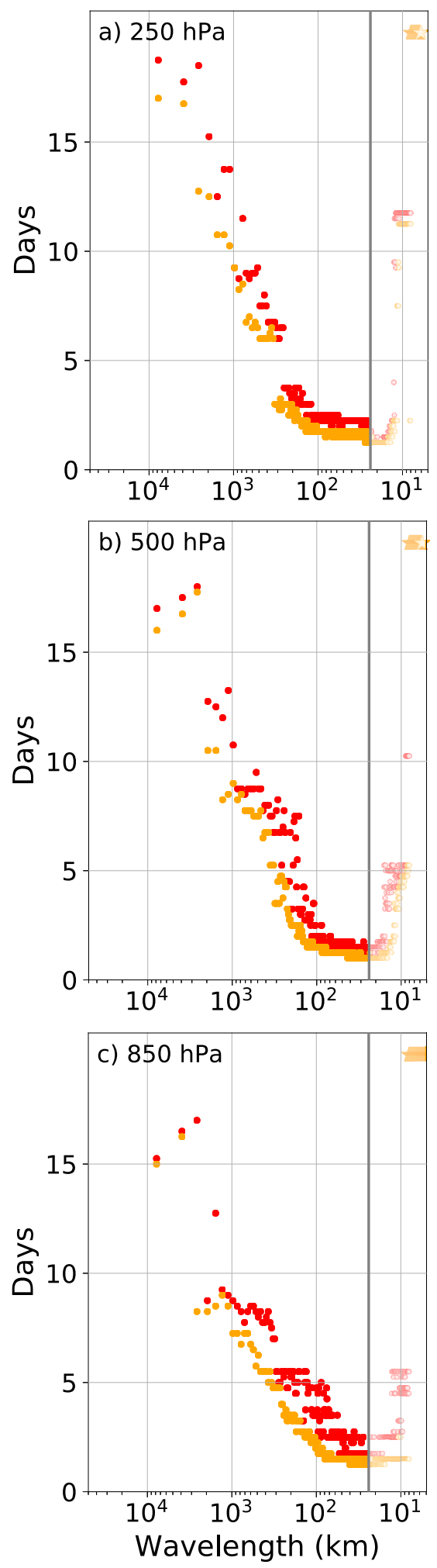

FIG. 11. Predictability limits computed in a land-free region over the South Pacific $\left(35^{\circ}-50^{\circ} \mathrm{S}, 85^{\circ} \mathrm{W}-180^{\circ}\right)$. are $<500 \mathrm{~km}$ ), and they evolve more slowly. The features are decidedly cold core, with 700-hPa temperatures directly under the vorticity maxima up to $5 \mathrm{~K}$ colder than the environment (not shown).

One TPV-like feature, marked with arrows in Fig. 13, develops when a lobe of vorticity is ejected from the middle latitudes into the arctic near $120^{\circ} \mathrm{W}$ on 28 October (simulation day 3 counting from 25 October 2012; Figs. 13a,b). This vortex can be traced as a coherent feature in both the control and 15-day perturbed run for at least 10 days. It tracks eastward along the poleward edge of the Canadian Arctic Archipelago, and at day 8, it is located at the northeastern tip of Ellesmere Island (Figs. 13c,d). Given that the scale of the vortex is only 200-300 km, the good agreement between control and perturbed run at day 8 is quite remarkable and unlike what we might expect when consulting Fig. $8 \mathrm{c}$, that is, a complete loss of predictability for features with a scale of $300 \mathrm{~km}$ after 6-7 days. The vortex continues to move eastward for another couple of days, and at simulation day 10, it is located near Spitsbergen (Figs. 13e,f). Shortly after this time, the feature begins to interact with the middle-latitude flow and loses its definition in both simulations.

TPVs may be relatively immune to error growth, since they draw their energy from radiation and not from convection or baroclinic processes (Cavallo and Hakim 2013). This would explain why the tracked features in Fig. 13 evolve very much alike in both the control and perturbed run up to the point where they begin to interact with the middle-latitude flow. A word of caution is needed, however, regarding the generality of the result. Figure 13d, for example, depicts a TPV-like vortex near the North Pole that has no "sibling" in the control run, suggesting that the predictability of TPV formation may be not as high, especially when the features are initiated by vorticity incursions from the middle latitudes. An indepth object-based predictability analysis of TPVs is necessary to draw more definite conclusions about their predictability.

\section{Predictability of convectively coupled equatorial waves}

Convectively coupled equatorial waves (hereafter simply equatorial waves) are one of the dominant sources of variability on weather time scales in the tropics (Kiladis et al. 2009), yet up to this day numerical weather prediction models struggle to simulate these phenomena. More specifically, operational models are not able to capture the propagation of waves, a problem that leads to unrealistically stationary rainfall patterns as seen in Fig. 1 of Dias et al. (2018). One of the goals 

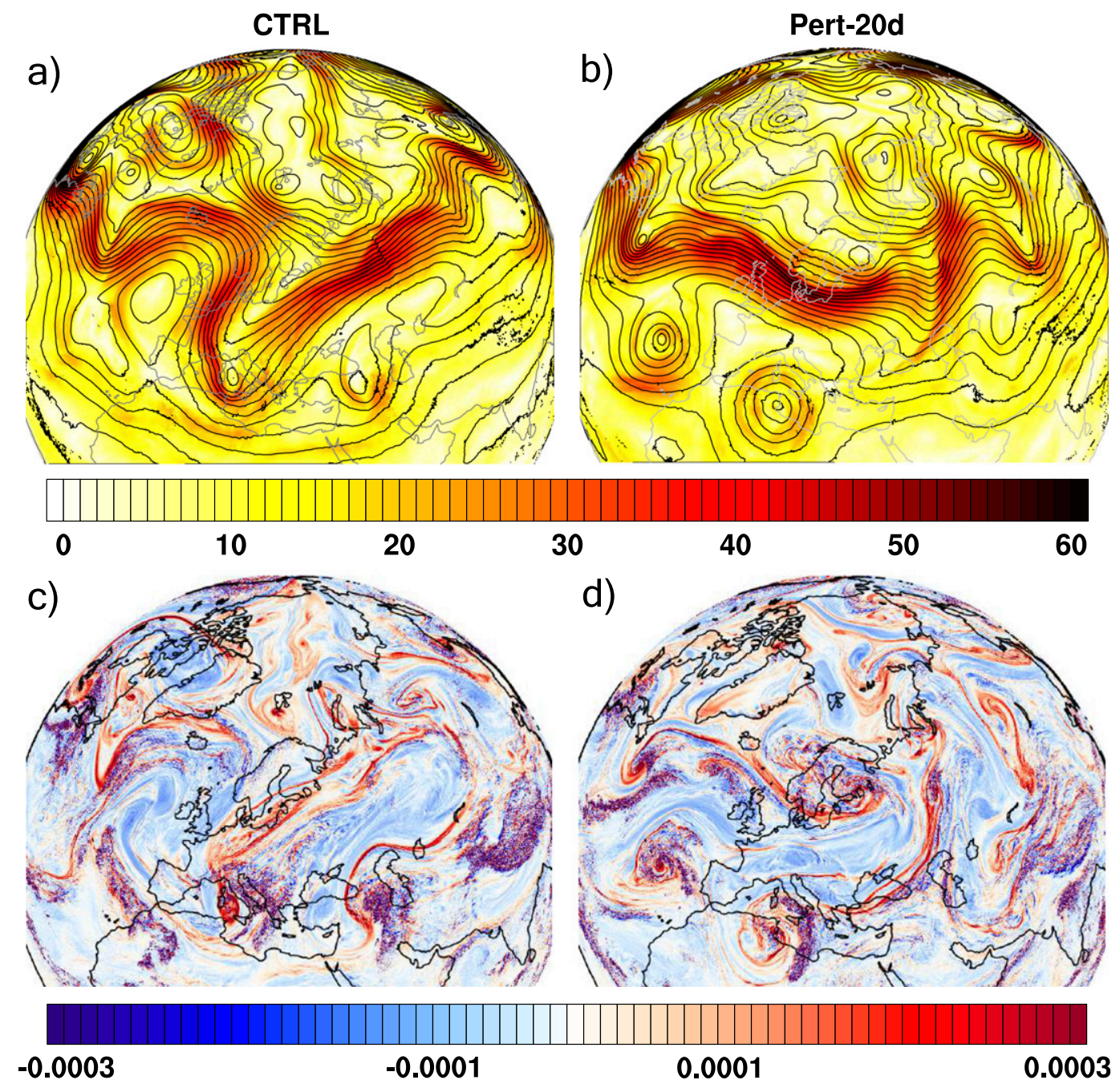

FIG. 12. Snapshots of middle-/upper-tropospheric flow in the (a),(c) control and (b),(d) 20-day perturbed run on simulation day 15 (0000 UTC 4 Nov 2012). Satellite view with a nadir point at $50^{\circ} \mathrm{N}, 25^{\circ} \mathrm{E}$ provides a look at the northern middle latitudes and Arctic region. (top) The 500-hPa wind speed (color shading; $\mathrm{m} \mathrm{s}^{-1}$ ) and Z500 height (contours) and (bottom) the 500-hPa relative vorticity $\left(\mathrm{s}^{-1}\right)$.

of this section is to show that storm-resolving models can mitigate this problem. Moreover, this section intends to 1) shed light on the error growth characteristics of equatorial waves and 2) develop the notion that equatorial waves play a role in the predictability of the tropics. Note that the spatial scale of some wave types (about $5000 \mathrm{~km}$ ) coincides with wavelengths that remain predictable beyond 20 days (Fig. 5), foreshadowing a possible relationship between equatorial waves and tropical predictability.

In contrast to the stationary patterns of current operational models (Dias et al. 2018), the 4-km MPAS generates rainfall patterns that propagate zonally throughout the 20-day simulation period (Fig. 14, shading). Tests with lower-resolution versions of MPAS revealed that explicit convection seems to be key to capture this propagation; specifically, a $7.5-\mathrm{km}$ MPAS with explicit convection showed rainfall patterns similar to the ones in Fig. 14, whereas a 15-km MPAS with parameterized convection produced substantially degraded rainfall patterns (not shown). The east- and westward-propagating rainfall features are reflecting various equatorial wave types, which can be identified through filtering. From here on, we will focus on Kelvin waves (eastward-propagating; Fig. 14a), Rossby waves (westward-propagating; Fig. 14a), mixed Rossby-gravity waves (westward-propagating, Fig. 14b), and westwardpropagating $n=1$ inertio-gravity waves (Fig. 14b). The amplitude of the waves is related to their spatial scale, with large-scale Kelvin waves being the most dominant 


\section{CTRL}
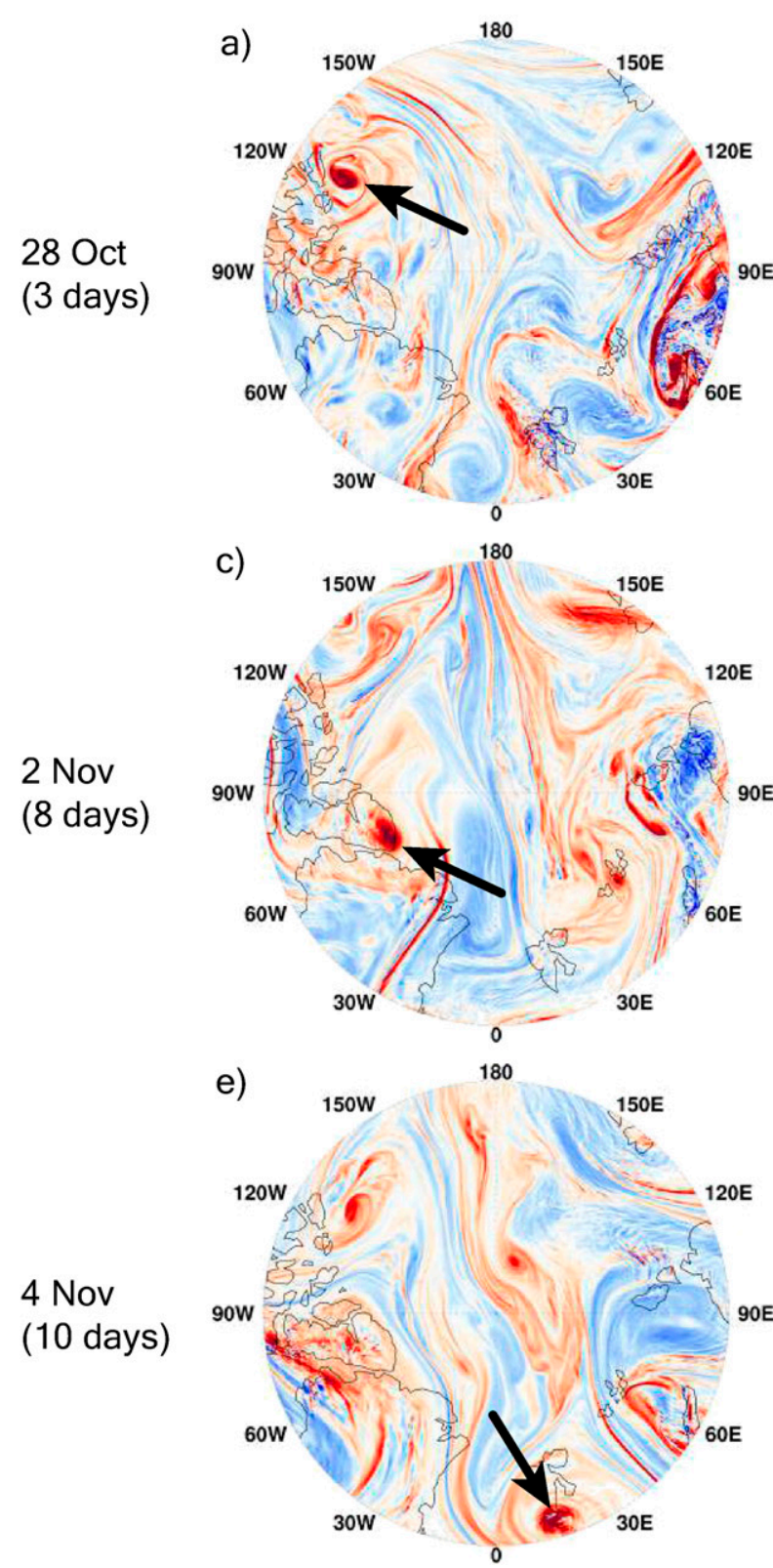

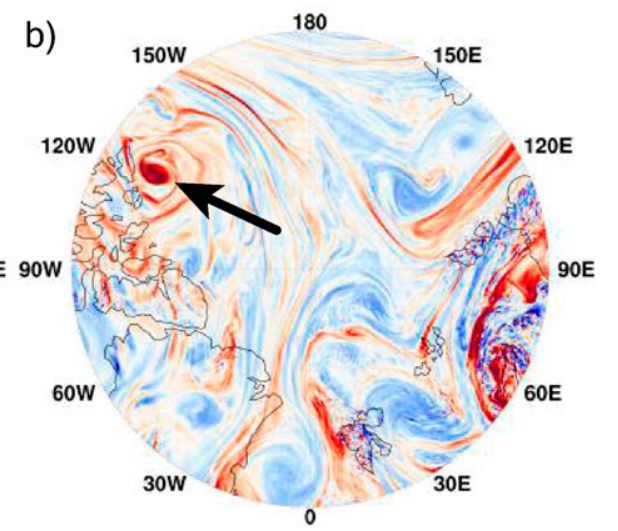

d)
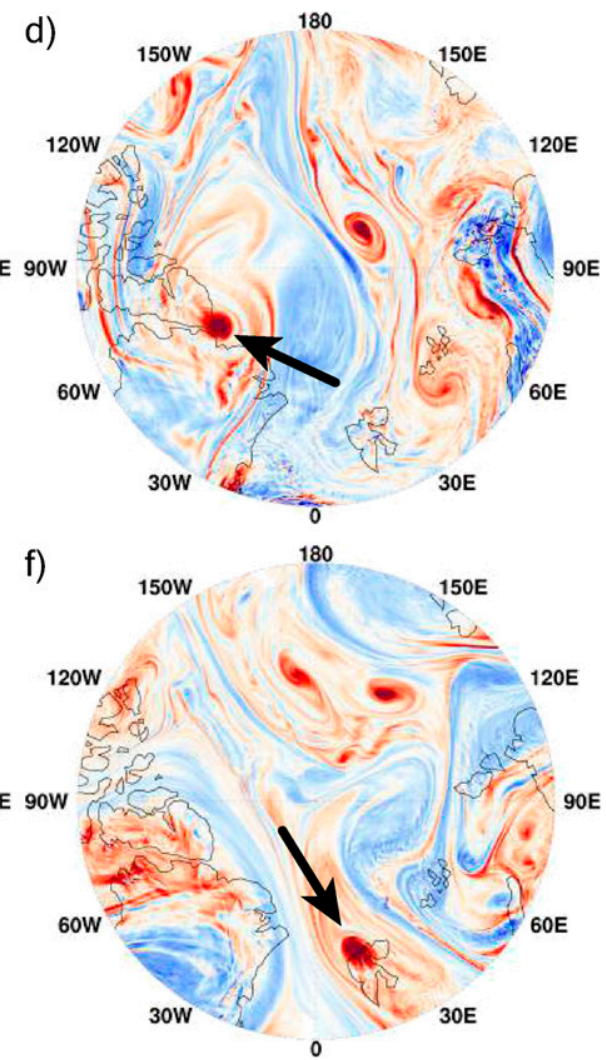

FIG. 13. Polar stereographic view of the northern polar cap, showing the evolution of 500-hPa relative vorticity in the (a),(c),(e) control and (b),(d),(f) 15-day perturbed run. Black arrows point to a vorticity feature that resembles a tropopause polar vortex. Color scale is as in Figs. 12 (bottom).

and small-scale inertio-gravity waves being the weakest (Fig. 14).

To be consistent with the rest of the study, the error growth analysis of equatorial waves will be based on the wave's wind fields. The waves leave a clear imprint on the low-level winds, and as in the rainfall fields, the trained eye can identify zonally propagating features even in the unfiltered wind fields (Fig. 15, shading).
Again, Kelvin waves have the most robust signal with a peak amplitude of $>3 \mathrm{~m} \mathrm{~s}^{-1}$ in the zonal wind (Fig. 15, top). Mixed Rossby-gravity waves, which leave a clear signal in the meridional wind component, are particularly abundant in the Indian Ocean and central Pacific (Fig. 15, bottom). Inertio-gravity waves occur more intermittently and have much weaker amplitudes, rendering them barely noticeable in the raw fields. The relative 


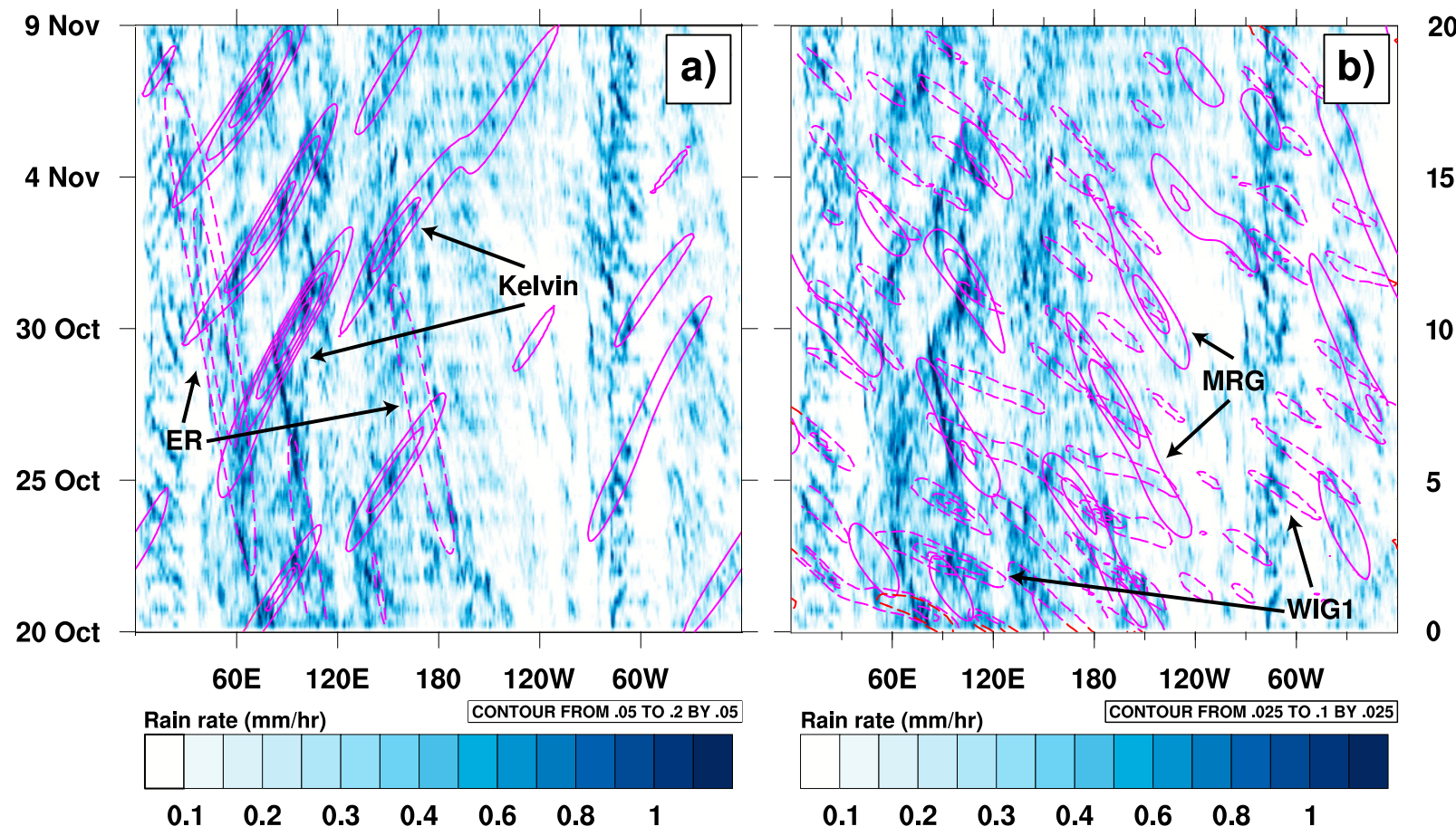

FIG. 14. Hovmöller plots of rainfall rate averaged between $10^{\circ} \mathrm{S}$ and $10^{\circ} \mathrm{N}$ (blue shading; $\mathrm{mm} \mathrm{h}^{-1}$ ), overlaid with contours that represent the filtered rainfall components of (a) Kelvin and equatorial Rossby (ER) waves (contoured every $0.05 \mathrm{~mm} \mathrm{~h}^{-1}$ ) and (b) mixed Rossby-gravity (MRG) and $n=1$ westward inertio-gravity (WIG1) waves (contoured every $0.025 \mathrm{~mm} \mathrm{~h}^{-1}$ ).

weakness of this wave type in the simulations agrees with observational studies (e.g., Wheeler and Kiladis 1999).

We will begin to investigate the predictability of the waves by determining how the wind patterns differ between the simulations. At first glance, the wind fields in the control and perturbed simulations are almost identical, including the wave-filtered wind components (Fig. 15). For example, amplitude and phase differences of the Kelvin waves are minor even at later simulation times, and similarly for mixed Rossby-gravity waves. The agreement between the raw wind patterns and filtered wave components in Fig. 15 is a sign that equatorial waves do not promote rapid error growth.

To assess error growth in the waves more quantitatively, we first survey the overall activity of each wave type and compare the waves' amplitude and phase between the control and 20-day perturbed run (Fig. 16, left two columns). Echoing Fig. 15, the two simulations show similar patterns of Kelvin, Rossby, and mixed Rossby-gravity waves with only minor differences. Consequently, the error is comparatively small when compared to the amplitude of the waves (Fig. 16, third column). Especially in the case of Kelvin and mixed Rossby-gravity waves, the error remains insignificant for at least a week (Figs. 16c,k). In comparison to the large-scale waves, errors in inertio-gravity waves grow faster, and the error magnitude becomes comparable to the magnitude of the actual waves by about day 10 (Fig. 16o).

The last step of the analysis is to assess the predictability of the waves, in this case by evaluating time series of normalized error KE (Fig. 16, rightmost column, note that time is on the $y$ axis). The error is normalized by the saturation limit, which was defined here as the background energy associated with each wave type:

$$
\mathrm{Limit}_{\mathrm{Wave}}(t)=2 \times \frac{1}{2}\left[\mathrm{KE}(t)_{\mathrm{Wave}}^{\mathrm{Ctrl}}+\mathrm{KE}(t)_{\mathrm{Wave}}^{\mathrm{Pert}}\right]
$$

Limit $_{\text {Wave }}$ is time dependent, which is intentional because in this way the normalization suppresses error magnitude fluctuations due to the time-varying amplitude of the waves. We first note that the error in the three large-scale waves does not saturate within 20 days (Figs. 16d,h,l). In fact, the error reaches only $50 \%$ saturation in mixed Rossby-gravity and $60 \%$ saturation in Kelvin and Rossby waves. On the other hand, the error in inertio-gravity waves saturates on day 18. In Kelvin and mixed Rossby-gravity waves, the error does not reach $10 \%$ saturation until day 10 , highlighting the resistance of equatorial waves to error growth.

The high predictability of large-scale equatorial waves begs the question of how predictable phenomena 
U850 (Kelvin, ER)
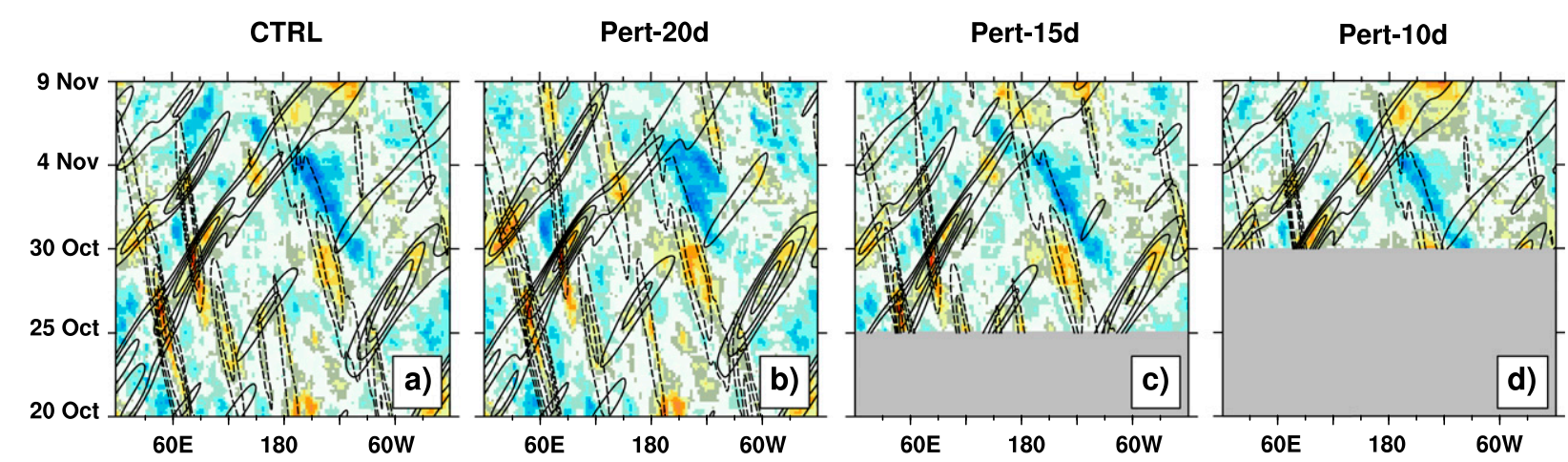

V850 (MRG, WIG1)
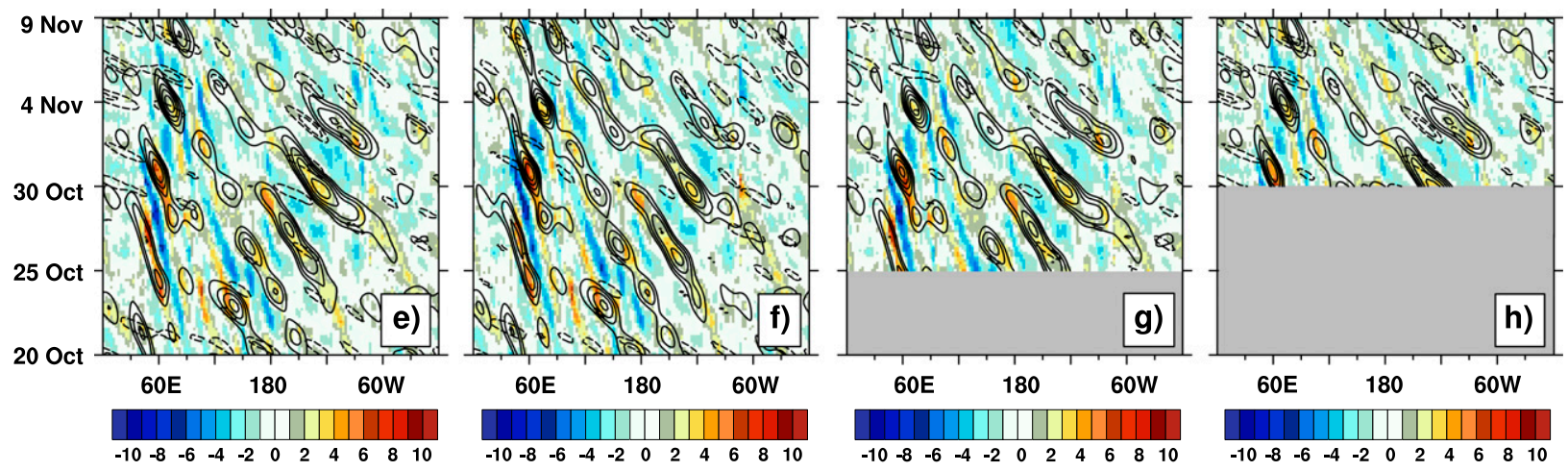

FIG. 15. Hovmöller plots of (a)-(d) 850-hPa zonal wind and (e)-(h) 850-hPa meridional wind (shading; $\mathrm{m} \mathrm{s}^{-1}$ ), overlaid with contours that represent the filtered wind components of (top) Kelvin and equatorial Rossby waves (contours from 1 to $2.5 \mathrm{~m} \mathrm{~s}^{-1}$, every $0.5 \mathrm{~m} \mathrm{~s}^{-1}$ ) and (bottom) mixed Rossby-gravity and $n=1$ westward inertio-gravity waves (contoured every $0.25 \mathrm{~m} \mathrm{~s}^{-1}$, starting at $0.5 \mathrm{~m} \mathrm{~s}^{-1}$ ). (left to right) The control, 20-day perturbed run, 15-day perturbed run, and 10-day perturbed run.

are that encompass even larger scales, such as the Madden-Julian oscillation (MJO). A comprehensive MJO predictability analysis is beyond the scope of this study, but as a first step, we will address the predictability of large-scale precipitation systems by comparing their evolution in the 20-day twins. Here, large-scale precipitation systems are identified with the large-scale precipitation tracking (LPT) method of Kerns and Chen (2016). The LPT method tracks contiguous areas within which the spatially smoothed rain rate exceeds $12 \mathrm{~mm}$ day $^{-1}$ (the rain rate itself is derived from 3-day rainfall accumulations).

October-November 2012 saw widespread convective activity over the Indo-Pacific warm pool associated with a low-amplitude MJO event ${ }^{3}$ (Fig. 17, shading). At simulation day 5, the LPT algorithm identifies three precipitation systems in each run, with no discernible differences in the size, shape, and location of these

\footnotetext{
${ }^{3}$ See historic MJO events at http://www.bom.gov.au/climate/mjo/.
}

features (Figs. 17a,b). Five days later, the westernmost precipitation system has disappeared in both runs, but the contours of the remaining system are still congruent, indicating that large-scale tropical precipitation features have high predictability up to at least day 10 (Figs. 17c,d).

By day 15 , the size of the precipitation systems has started to differ between the runs, indicating growing differences in the intensity and location of rainfall (Figs. 17e,f). A new system has developed over the Malay Peninsula, yet the area of this system is much larger in the perturbed run than in its counterpart. Overall, precipitation in the domain reduces by day 20 , and only one object exists that qualifies as large-scale precipitation system (Fig. 17g). This system is associated with a cyclone tracking into the Bay of Bengal. The tropical cyclone exists in the perturbed simulation as well, but it is weaker and taking a more westward track (Fig. 17h).

Note that despite differences in the size and shape of large-scale precipitation systems by day 15 , the overall 
CTRL
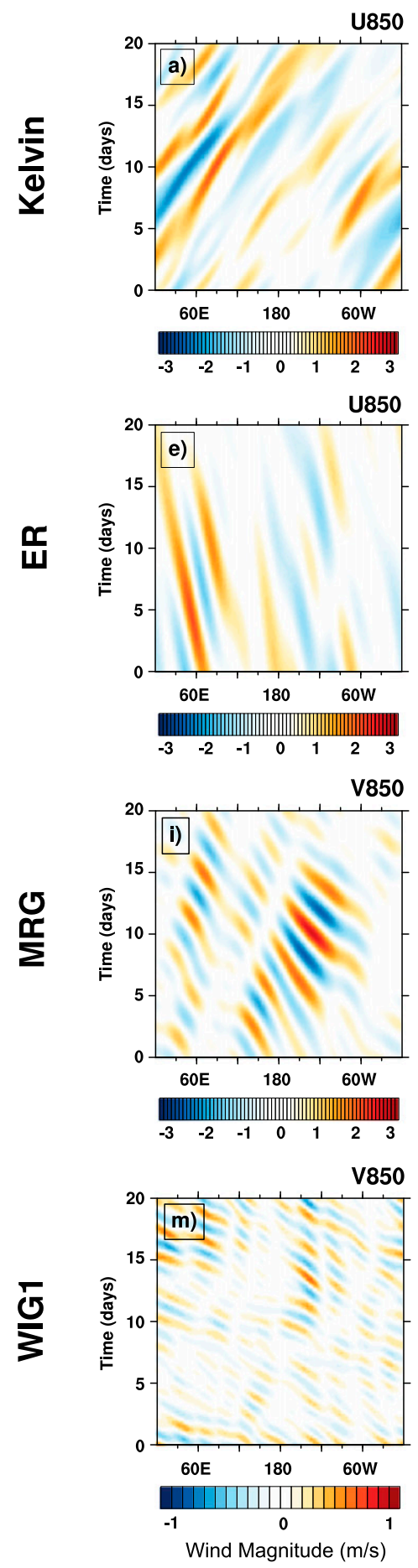

Pert-20d
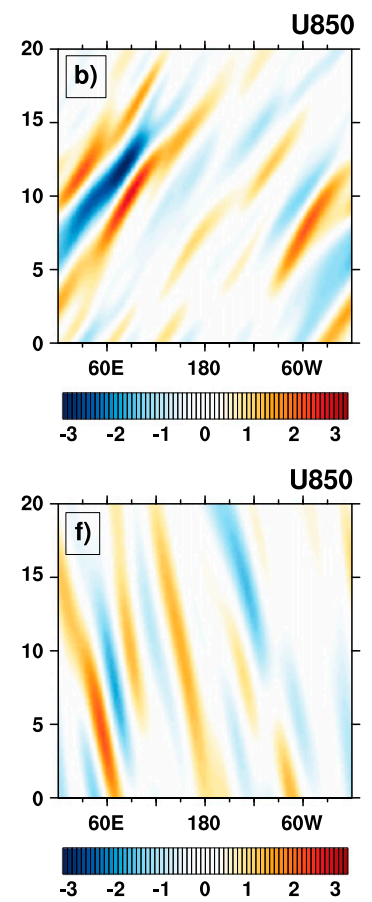

V850

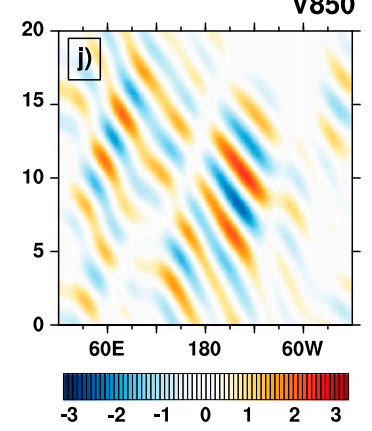

V850

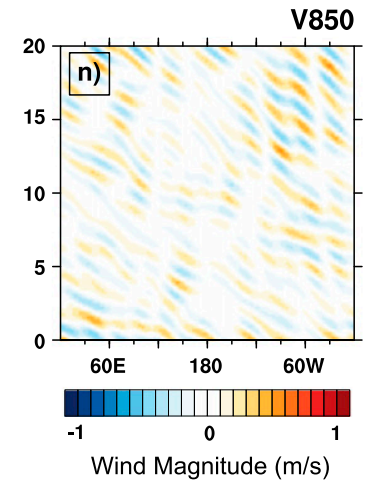

Error
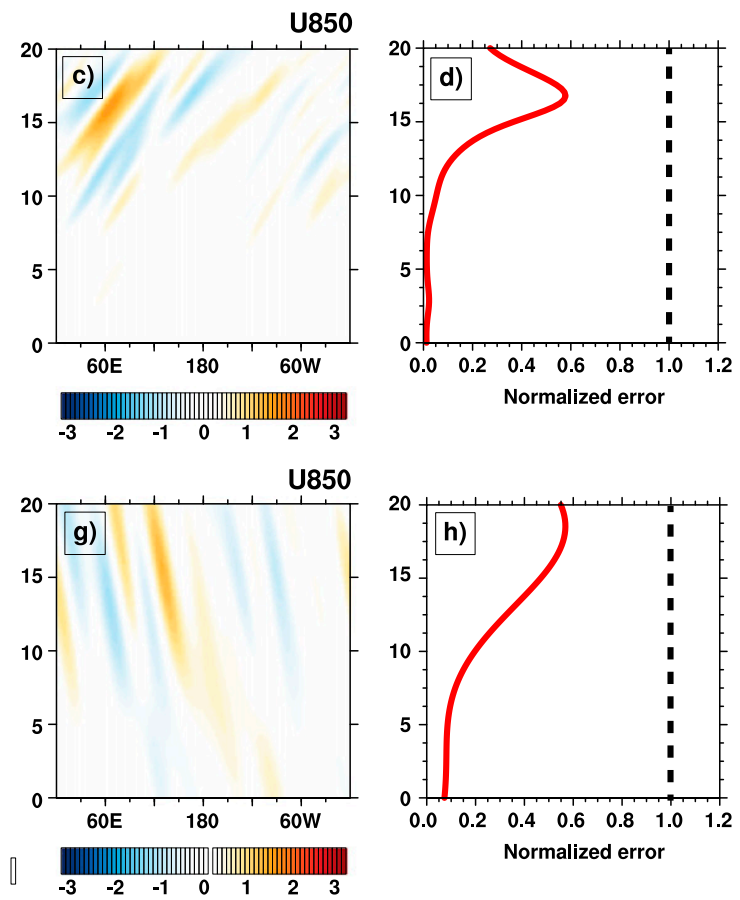

V850
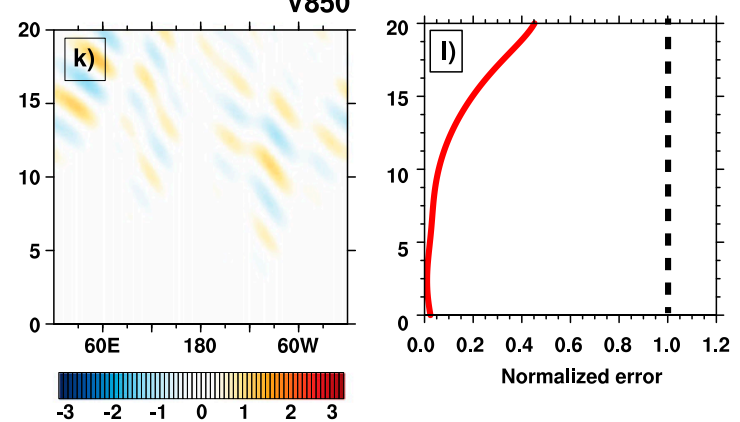

V850
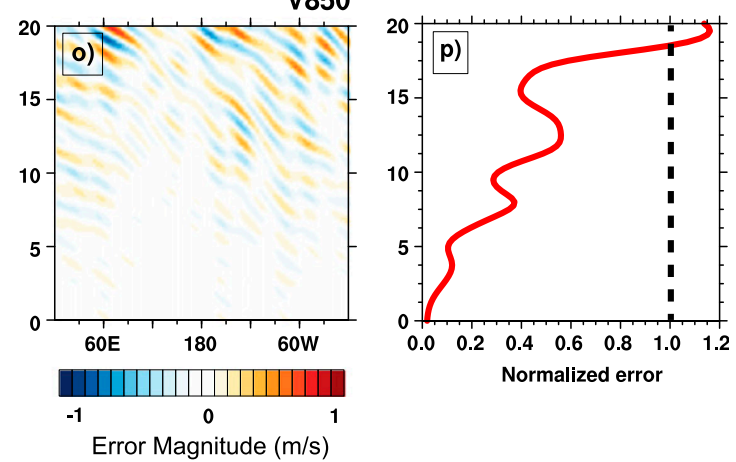

FIG. 16. Hovmöller plots of wave-filtered winds from the (left) control and (left center) 20-day perturbed run ( $\mathrm{m} \mathrm{s}^{-1}$ ), (right center) difference between the two ("error"; $\mathrm{m} \mathrm{s}^{-1}$ ), and (right) time series of the error. The error has been normalized by the saturation magnitude (dashed line), which was computed here as sum of the wave KE from the control and perturbed runs. Rows show the different wave types: (a)-(d) Kelvin, (e)-(h) equatorial Rossby, (i)-(l) mixed Rossby-gravity, and (m)-(p) $n=1$ westward inertio-gravity waves. 


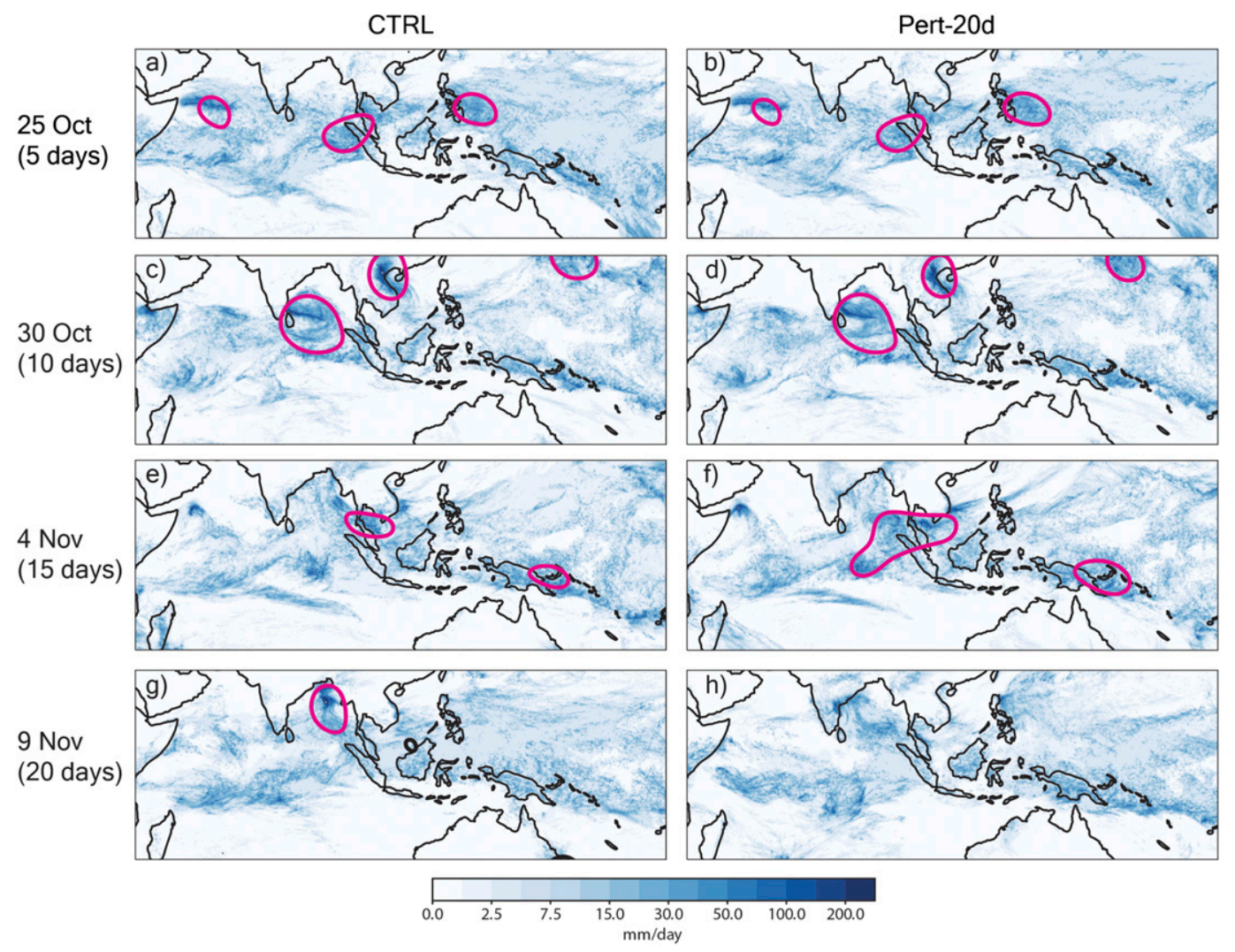

FIG. 17. Average rain rate for the 3 days ending at the indicated times (color shaded) and large-scale precipitation systems (contours), here defined as areas within the $12 \mathrm{~mm}_{\text {day }}{ }^{-1}$ contour of the spatially smoothed rain-rate field [for specifics, see Kerns and Chen (2016)].

basin-scale rainfall distribution remains similar in the two runs (Figs. 17e-h, shading). Specifically, the equatorial eastern Indian Ocean region becomes progressively drier, and at day 20, Indian Ocean rainfall is concentrated in an ITCZ band south of the equator. One possible reason for this similarity is that both runs use identical SST fields. The prescribed SSTs tend to anchor convection in certain regions and may therefore artificially enhance predictability on very large scales. Future studies with a coupled atmosphere-ocean model will be helpful to shed light on this issue.

In summary, the predictability of equatorial waves depends on the wave type, and large-scale waves seem to be predictable for $>20$ days. This finding agrees well with Ying and Zhang (2017), who used a limited-area model. The relatively long predictability of equatorial waves provides an explanation for why the tropics have longer predictability than the extratropics. The notion that equatorial waves determine the predictability of the tropics is substantiated by another fact: recall that the error growth rate in the tropics featured an uptick around day 10 (Fig. 4) — essentially the same time at which the error in equatorial waves begins to grow noticeably
(Figs. 16d,h,l). Large-scale precipitation system associated with the MJO are predictable on time scales similar to equatorial waves, and the basin-scale rain fall pattern seems to be insensitive to error growth even at 20 days; however, this finding may be an artifact of using prescribed SSTs.

\section{Summary and conclusions}

The overall goal of this study was to better understand and quantify atmospheric predictability. The objectives were to 1) compare error growth between the tropics, middle latitudes, and polar regions, 2) quantify the intrinsic predictability of each latitude zone, and 3) explore the predictability of equatorial waves. The analysis was facilitated with output from the global storm-resolving simulations of Judt (2018).

The main findings of the present study can be summarized as follows:

- Error growth is latitude dependent and consistent with the underlying atmospheric dynamics. During the first couple of hours, the error magnitude was highest in 
the tropics and lowest in the polar regions. However, error growth rates in the extratropics soon become larger than in the tropics and lead to faster destruction of predictability (Figs. 2 and 5).

- The tropical atmosphere has longer predictability than the extratropical atmosphere. In this particular experiment, the error in the middle latitudes and polar regions saturated after 17 days, whereas the error in the tropics did not saturate during the 20-day experiment period (Fig. 2).

- A canonical KE spectrum with logarithmic slopes of -3 at the synoptic scales and $-5 / 3$ at the mesoscales exists only in the middle latitudes. In the tropics and polar regions, the slopes are more uniform (Fig. 7). In other words, the famous spectrum of Nastrom and Gage (1985) does not seem to be universal, and it exists in a pure form only in the middle-latitude upper troposphere (Fig. 7b). The latitude dependence of the spectral slope-and, by extension, the latitude dependence of error growth-can be explained by differences in the underlying dynamics.

- The predictability limit of atmospheric motions is a complex function of scale, height, and latitude (Fig. 8).

- Errors in equatorial Kelvin, Rossby, and mixed Rossby-gravity waves did not saturate during the 20-day experiment period (Fig. 16). This finding provides an explanation for why the tropics have longer predictability. More specifically, equatorial waves seem to be more resistant to error growth than baroclinic systems.

The finding that tropical predictability exceeds that of the extratropics supports the results of Straus and Paolino (2008), but somewhat contradicts conventional wisdom and current numerical weather prediction experience (e.g., Dias et al. 2018). This apparent contradiction may be a reflection of the discrepancy between practical and intrinsic predictability (Melhauser and Zhang 2012). Specifically, tropical predictability may currently be more limited due to model error than due to the growth of internal errors. Given that the parameterization of convection is one of the largest sources of model error (Randall et al. 2003), future generations of global storm-resolving models may be able to exploit the predictability of the tropics more effectively.

A positive conclusion of this study is that current numerical weather prediction procedures have not yet reached the limits of predictability, even though the rate of forecast skill improvement has slowed in recent years. The studies of Selz (2019) and Zhang et al. (2019) arrived at similar conclusions; however, they only examined the predictability of the middle latitudes. Specifically, they argue that the predictability horizon of middle-latitude weather can be extended by up to 5 days. The present study suggests that there is even more potential the tropics. It is up to the meteorological community to exploit this predictability by improving the models, data assimilation techniques, and observational capabilities.

Acknowledgments. The simulations for this study were generated with generous help from Michael Duda (NCAR). Bill Skamarock (NCAR) assisted in designing the project and determining the computational resources. Rich Rotunno and Rosimar Rios-Berrios (both NCAR) provided informal review of this manuscript, and the comments from three anonymous reviewers helped to further improve the paper. High-performance computing support from Yellowstone (ark:/85065/d7wd3xhc) provided by NCAR's Computational and Information Systems Laboratory, sponsored by the National Science Foundation, is greatly acknowledged. The National Center for Atmospheric Research is sponsored by the National Science Foundation.

\section{REFERENCES}

Baldwin, M. P., and T. J. Dunkerton, 2001: Stratospheric harbingers of anomalous weather regimes. Science, 294, 581-584, https://doi.org/10.1126/science.1063315.

Baumgart, M., P. Ghinassi, V. Wirth, T. Selz, G. C. Craig, and M. Riemer, 2019: Quantitative view on the processes governing the upscale error growth up to the planetary scale using a stochastic convection scheme. Mon. Wea. Rev., 147, 1713-1731, https://doi.org/10.1175/MWR-D-18-0292.1.

Bengtsson, L., and K. I. Hodges, 2006: A note on atmospheric predictability. Tellus, 58A, 154-157, https://doi.org/10.1111/ j.1600-0870.2006.00156.x.

Boer, G. J., 1994: Predictability regimes in atmospheric flow. Mon. Wea. Rev., 122, 2285-2295, https://doi.org/10.1175/15200493(1994)122<2285:PRIAF>2.0.CO;2.

Buizza, R., and M. Leutbecher, 2015: The forecast skill horizon. Quart. J. Roy. Meteor. Soc., 141, 3366-3382, https://doi.org/ 10.1002/qj.2619.

Cavallo, S. M., and G. J. Hakim, 2009: Potential vorticity diagnosis of a tropopause polar cyclone. Mon. Wea. Rev., 137, 13581371, https://doi.org/10.1175/2008MWR2670.1.

$\longrightarrow$, and - 2010: Composite structure of tropopause polar cyclones. Mon. Wea. Rev., 138, 3840-3857, https://doi.org/ 10.1175/2010MWR3371.1.

$\longrightarrow$, and - 2013: Physical mechanisms of tropopause polar vortex intensity change. J. Atmos. Sci., 70, 3359-3373, https:// doi.org/10.1175/JAS-D-13-088.1.

Dias, J., M. Gehne, G. N. Kiladis, N. Sakaeda, P. Bechtold, and T. Haiden, 2018: Equatorial waves and the skill of NCEP and ECMWF numerical weather prediction systems. Mon. Wea. Rev., 146, 1763-1784, https://doi.org/10.1175/ MWR-D-17-0362.1.

Durran, D. R., and M. Gingrich, 2014: Atmospheric predictability: Why butterflies are not of practical importance. J. Atmos. Sci., 71, 2476-2488, https://doi.org/10.1175/JASD-14-0007.1. 
__ P. A. Reinecke, and J. D. Doyle, 2013: Large-scale errors and mesoscale predictability in Pacific Northwest snowstorms. J. Atmos. Sci., 70, 1470-1487, https://doi.org/10.1175/JAS-D12-0202.1.

Errico, R. M., 1985: Spectra computed from a limited area grid. Mon. Wea. Rev., 113, 1554-1562, https://doi.org/10.1175/15200493(1985)113<1554:SCFALA > 2.0.CO;2.

Grell, G. A., and S. R. Freitas, 2014: A scale and aerosol aware stochastic convective parameterization for weather and air quality modeling. Atmos. Chem. Phys., 14, 5233-5250, https:// doi.org/10.5194/acp-14-5233-2014.

Hersbach, H., and D. Dee, 2016: ERA5 reanalysis is in production. ECMWF Newsletter, No. 147, ECMWF, Reading, United Kingdom, 7, https://www.ecmwf.int/sites/default/files/elibrary/ 2016/16299-newsletter-no147-spring-2016.pdf.

Judt, F., 2018: Insights into atmospheric predictability through global convection-permitting model simulations. J. Atmos. Sci., 75, 1477-1497, https://doi.org/10.1175/JAS-D-17-0343.1.

Kerns, B. W., and S. S. Chen, 2016: Large-scale precipitation tracking and the MJO over the Maritime Continent and IndoPacific warm pool. J. Geophys. Res. Atmos., 121, 8755-8776, https://doi.org/10.1002/2015JD024661.

Kiladis, G. N., M. C. Wheeler, P. T. Haertel, K. H. Straub, and P. E. Roundy, 2009: Convectively coupled equatorial waves. Rev. Geophys., 47, RG2003, https://doi.org/10.1029/2008RG000266.

Lorenz, E. N., 1969: The predictability of a flow which possesses many scales of motion. Tellus, 21, 289-307, https://doi.org/ 10.3402/tellusa.v21i3.10086.

_ 1982: Atmospheric predictability experiments with a large numerical model. Tellus, 34, 505-513, https://doi.org/10.3402/ tellusa.v34i6.10836.

Mapes, B., S. Tulich, T. Nasuno, and M. Satoh, 2008: Predictability aspects of global aqua-planet simulations with explicit convection. J. Meteor. Soc. Japan, 86A, 175-185, https://doi.org/ 10.2151/jmsj.86A.175.

Melhauser, C., and F. Zhang, 2012: Practical and intrinsic predictability of severe and convective weather at the mesoscales. J. Atmos. Sci., 69, 3350-3371, https://doi.org/10.1175/ JAS-D-11-0315.1.

Menchaca, M. Q., and D. R. Durran, 2019: The influence of gravity waves on the slope of the kinetic energy spectrum in simulations of idealized midlatitude cyclones. J. Atmos. Sci., 76, 2103-2122, https://doi.org/10.1175/JAS-D-18-0329.1.

Nastrom, D. G., and K. S. Gage, 1985: A climatology of atmospheric wavenumber spectra of wind and temperature observed by commercial aircraft. J. Atmos. Sci., 42, 950-960, https://doi.org/ 10.1175/1520-0469(1985)042<0950:ACOAWS >2.0.CO;2.

Randall, D., M. Khairoutdinov, A. Arakawa, and W. Grabowski, 2003: Breaking the cloud parameterization deadlock. Bull. Amer. Meteor. Soc., 84, 1547-1564, https://doi.org/10.1175/ BAMS-84-11-1547.

Reynolds, C. A., P. J. Webster, and E. Kalnay, 1994: Random error growth in NMC's global forecasts. Mon. Wea. Rev., 122, 12811305, https://doi.org/10.1175/1520-0493(1994)122<1281:REGING> 2.0.CO;2.

Selz, T., 2019: Estimating the intrinsic limit of predictability using a stochastic convection scheme. J. Atmos. Sci., 76, 757-765, https://doi.org/10.1175/JAS-D-17-0373.1.
— and G. C. Craig, 2015: Simulation of upscale error growth with a stochastic convection scheme. Geophys. Res. Lett., $\mathbf{4 2}$, 3056-3062, https://doi.org/10.1002/2015GL063525.

Simmons, A. J., and A. Hollingsworth, 2002: Some aspects of the improvement in skill of numerical weather prediction. Quart. J. Roy. Meteor. Soc., 128, 647-677, https://doi.org/10.1256/ 003590002321042135 .

Skamarock, W. C., J. B. Klemp, M. G. Duda, L. D. Fowler, S.-H. Park, and T. D. Ringler, 2012: A multiscale nonhydrostatic atmospheric model using centroidal Voronoi tesselations and C-grid staggering. Mon. Wea. Rev., 140, 3090-3105, https:// doi.org/10.1175/MWR-D-11-00215.1.

Straus, D. M., and D. Paolino, 2008: Intermediate time error growth and predictability: Tropics versus mid-latitudes. Tellus 61, 579-586, https://doi.org/10.1111/j.1600-0870.2009.00411.x.

Sun, Y. Q., R. Rotunno, and F. Zhang, 2017: Contributions of moist convection and internal gravity waves to building the atmospheric 5/3 kinetic energy spectra. J. Atmos. Sci., 74, 185-201, https://doi.org/10.1175/JAS-D-16-0097.1.

Thompson, D. W. J., M. P. Baldwin, and J. M. Wallace, 2002: Stratospheric connection to Northern Hemisphere wintertime weather: Implications for prediction. J. Climate, 15, 1421-1428, https://doi.org/10.1175/1520-0442(2002)015<1421:SCTNHW > 2.0.CO;2.

Tribbia, J. J., and D. P. Baumhefner, 2004: Scale interactions and atmospheric predictability: An updated perspective. Mon. Wea. Rev., 132, 703-713, https://doi.org/10.1175/15200493(2004)132<0703:SIAAPA > 2.0.CO;2.

Tripathi, O. P., and Coauthors, 2016: Examining the predictability of the stratospheric sudden warming of January 2013 using multiple NWP systems. Mon. Wea. Rev., 144, 19351960, https://doi.org/10.1175/MWR-D-15-0010.1.

Weyn, J. A., and D. R. Durran, 2017: The dependence of the predictability of mesoscale convective systems on the horizontal scale and amplitude of initial errors in idealized simulations. J. Atmos. Sci., 74, 2191-2210, https://doi.org/10.1175/ JAS-D-17-0006.1.

Wheeler, M., and G. N. Kiladis, 1999: Convectively coupled equatorial waves: Analysis of clouds and temperature in the wavenumber-frequency domain. J. Atmos. Sci., 56, 374-399, https://doi.org/10.1175/1520-0469(1999)056<0374:CCEWAO> 2.0.CO;2.

Ying, Y., and F. Zhang, 2017: Practical and intrinsic predictability of multiscale weather and convectively coupled equatorial waves during the active phase of an MJO. J. Atmos. Sci., 74, 3771-3785, https://doi.org/10.1175/JAS-D-17-0157.1.

Zhang, F., C. Snyder, and R. Rotunno, 2003: Effects of moist convection on mesoscale predictability. J. Atmos. Sci., 60 , 1173-1185, https://doi.org/10.1175/1520-0469(2003)060<1173: EOMCOM $>2.0 . \mathrm{CO} ; 2$.

$\longrightarrow$, N. Bei, R. Rotunno, C. Snyder, and C. C. Epifanio, 2007: Mesoscale predictability of moist baroclinic waves: Convectionpermitting experiments and multistage error growth dynamics. J. Atmos. Sci., 64, 3579-3594, https://doi.org/10.1175/JAS4028.1.

— , Y. Q. Sun, L. Magnusson, R. Buizza, S.-J. Lin, J.-H. Chen, and K. Emanuel, 2019: What is the predictability limit of midlatitude weather? J. Atmos. Sci., 76, 1077-1091, https:// doi.org/10.1175/JAS-D-18-0269.1. 